\title{
Vibrational and Electronic Spectra of 2-Phenyl-2-Imidazoline: A Combined Experimental and Theoretical Study
}

\author{
Yeddu Sushma Priya', Kokkiripati Ramachandra Rao², Pallavajhula Venkata Chalapathi \\ Adamilli Veeraiah ${ }^{*}$ \\ ${ }^{1}$ Department of Physics, Adikavi Nannaya University, Rajamahendravaram, India \\ ${ }^{2}$ Crystal growth and Nano Science Research Centre, Department of Physics, Government College (A), Rajamahendravaram, India \\ ${ }^{3}$ Department of Physics, University College of Engineering, Javaharlal Nehru Technological University, Guntur, India \\ ${ }^{4}$ Molecular Spectroscopy Laboratory, Department of Physics, DNR College (A), Bhimavaram, India \\ Email:*avru@rediffmail.com
}

How to cite this paper: Priya, Y.S., Rao, K.R., Chalapathi, P.V. and Veeraiah, A. (2018) Vibrational and Electronic Spectra of 2-Phenyl-2-Imidazoline: A Combined Experimental and Theoretical Study. Journal of Modern Physics, 9, 753-774.

https://doi.org/10.4236/jmp.2018.94049

Received: January 20, 2018

Accepted: March 27, 2018

Published: March 30, 2018

Copyright $\odot 2018$ by authors and Scientific Research Publishing Inc. This work is licensed under the Creative Commons Attribution International License (CC BY 4.0).

http://creativecommons.org/licenses/by/4.0/

\begin{abstract}
The structural properties and vibrational frequencies of 2-phenyl-2-imidazoline have been investigated using theoretical and techniques by which a good correlation was observed. The assignments of the vibrational modes were performed with the help of normal co-ordinate analysis following the Scaled Quantum Mechanical Force Field methodology. Natural bond orbital analysis and the highest occupied molecular orbital-lowest unoccupied molecular orbital gap analysis have been carried out. UV-visible spectrum of the compound was recorded and compared with the theoretical UV-visible spectrum of the title molecule using Symmetry Adapted Cluster-Configuration Interaction method which yielded good agreement. Our results reflect that the title compound can be used as good source of UV light.
\end{abstract}

\section{Keywords}

2-Phenyl-2-Imidazoline, DFT, FT-IR, FT-RAMAN, NBO, HOMO and LUMO

\section{Introduction}

At present, there is an enormous deal of scientific attention in the field of Imidazoline derivatives. Imidazoline is a nitrogen compound and it can be utilized in organic dyes as nitrogenous electron donors since it introduces additional electron donors at position 2. Because of high molar extinction coefficient, Imidazoline and other molecular structures containing nitrogen developed as metal 
free organic dyes. Usually, metal-free organic dyes have the apparent molecular structure of the electron donor element and the acceptor element connected by the conjugated chain $(\mathrm{D}-\pi-\mathrm{A}) .2$-imidazoline is a one of the isomers derived from the imidazole. Imidazoline derivatives are pharmaceutically and biologically very significant. Imidazolines exhibit major pharmacological and biological activities such as antidepressive [1], antihyperglycemic [2], antihypercholesterolemic [3], anti-inflammatory [4] and antihypertensive [5]. The drug clonidine containing Imidazoline is used independently otherwise it is used with the combination of other medications for the therapy of high blood pressure. Imidazoline and its derivatives are important sort of inhibitors which could effectively inhibit corrosion of carbon steel against $\mathrm{CO}_{2}$ and $\mathrm{H}_{2} \mathrm{~S}$ [6] [7]. Recently C.D. Contreras et al. [8] reported the structural and vibrational analyses of 2-(2-benzofuranyl)-2-imidazoline.

The purpose of this study is to obtain the theoretical information about the molecular structure and electronic parameters of 2-phenyl-2-imidazoline. Density functional theory has been adopted for the precise study of the molecular geometry and electronic distribution. Here we study the experimental and theoretical study of the 2-phenyl-2-imidazoline for the first time.

\section{Experimental Studies}

The powder sample of 2-phenyl-2-imidazoline (here after termed as 2PI) has been procured from sigma Aldrich chemical company (USA) with a confirmed purity greater than $98 \%$ and was utilized in the consequent spectroscopic study with no any additional purification.

\subsection{FT-IR Spectrum}

Fourier transform infrared spectrum of the $2 \mathrm{PI}$ is recorded at the temperature $302.15^{\circ} \mathrm{K}$ in the region $4000-400 \mathrm{~cm}^{-1}$ by Nicolet 6700 FTIR spectrometer fitted by means of a Thermo Nicolet Continuum IR microscope and through a Renishaw in via., Raman microscope with UV or visible laser excitation at a resolution of $\pm 1 \mathrm{~cm}^{-1}$. FTIR spectrum of the given compound was recorded by $\mathrm{KBr}$ pellet method with Spectrum GX Fourier transform-infrared spectroscopy (FT-IR) spectrometer.

\subsection{FT-Raman Spectrum}

The FT-Raman spectrum of the molecule was recorded at a $4 \mathrm{~cm}^{-1}$ resolution through a Nicolet Magna 750 Raman spectrometer functioned with an InGaAs (Indium Gallium Arsenide) semiconductor detector in the $3500-0 \mathrm{~cm}^{-1}$ region. The excitation source utilized was the 1064-nm line from Neodymium: Yttrium Aluminum Garnet laser. The laser power at the sample location was typically $500 \mathrm{~mW}$.

\subsection{UV-Vis Spectrum}

UV-Vis spectrum of the 2PI has been recorded in the region of $200-400 \mathrm{~nm}$ with a Perkin Elmer Lambda 35 UV-vis spectrometer. All the data were recorded 
after 1 cycle, with a period of $1 \mathrm{~nm}$, slit width of $2 \mathrm{~nm}$ and scan rate of 240 $\mathrm{nm} \cdot \mathrm{min}^{-1}$ by the spectral resolution of $0.05-4.0 \mathrm{~nm}$. The slit width was situating to $15 \mathrm{~nm}$ for the emission monochromator and to $10 \mathrm{~nm}$ for the excitation monochromator.

\section{Computational Details}

Quantum chemical density functional computations on 2PI were carried out using Becke's three-parameter (B3LYP) hybrid DFT level employed to optimize the molecular geometry through the $6-31 \mathrm{G}(\mathrm{d}, \mathrm{p})$ basis set using Gaussian $09 \mathrm{~W}$ Revision-A.02 SMP [9], Gauss view 5.0.8 molecular visualization program package [10]. The vibrational frequencies are computed through the second order derivative of the energy with $6-311++G^{* *}$ basis set. In this study, it is noticed that the theoretically computed frequencies were slightly more than the experimentally observed frequencies. For a better match between the calculated and experimental frequencies, scaling is carried out on the calculated frequencies. Scaling was executed in accordance with scaled Quantum mechanical (SQM) process [11] [12] [13]. The depictions of the calculated frequencies all over the scaling process were followed by means of the potential energy distribution (PED) matrix. The classification of the normal modes through potential energy distribution (PED) was performed through the MOLVIB -7.0 Program written by Prof. T. Sundius [14] [15]. Natural bond orbital (hereafter, it is abbreviated as NBO) calculations [16] were performed to recognize the hyper conjugation or intra-molecular delocalization with the implementation of NBO 3.1 program in the Gaussian 09W Revision-A.02 SMP version [9] package at the DFT/B3LYP level.

\subsection{The Prediction of Raman Intensities}

The Raman activity ( $\left.S_{i}\right)$ computed by Gaussian 09W Revision-A.02 SMP version [9] and altered through scaling process with MOLVIB were modified to relative Raman intensity $\left(I_{i}\right)$ by the subsequent equation as of the basic theory of Raman scattering [17] [18].

$$
I_{i}=\frac{f\left(v_{0}-v_{i}\right)^{4} S i}{v_{i}\left(1-\exp \left(\frac{-h c v_{i}}{k T}\right)\right)}
$$

Here $v_{i}$ is the normal mode vibrational wave number; $v_{0}$ is the exciting frequency (in $\mathrm{cm}^{-1}$ units); $h, c$ and $k$ are the universal constants and $f$ is appropriately chosen common normalization factor used for all the intensities. Pure Lorentzian band shapes were employed to draw simulated FTIR and FT-Raman spectra.

\section{Results and Discussion}

\subsection{Molecular Geometry}

The title compound $2 \mathrm{PI}$ has a non-planar structure with $\mathrm{C}_{1}$ symmetry, 21 atoms 
and therefore it has 57 normal modes of internal vibrations. The vibrational harmonic frequencies of the given molecule are computed with Density Functional Theory with Becke-3-Lee-Yang-Parr (B3LYP) method [19]. The optimized structure parameters of 2PI computed by DFT/B3LYP level by means of 6-31G $(\mathrm{d}, \mathrm{p})$ basis set are represented in Table 1 in accordance with the atom numbering given in Figure 1. The potential energy surface (PES) scan for C3-C4-C5-H15 was detected by varying the torsion angle for each $10^{\circ}$ up to $360^{\circ}$ rotation around the bond using $6-31 \mathrm{G}(\mathrm{d}, \mathrm{p})$ basis set. The graph between the potential energy and the dihedral angle was represented in Figure 2. The minimum energy acquired from potential energy surface scan was 454.9756695 Hartrees.

The theoretically calculated optimized geometrical parameters were compared with the single crystal XRD data of 2-[2-(2-Hydroxyethoxy) phenyl]-4, 4, 5, 5-tetramethyl-2-imidazoline-1-oxyl 3-oxide [20] data since the crystal data of the title molecule is not available. It was studied that here a few differences in the investigational and calculated geometrical parameters. These differences might

Table 1. Optimized geometrical parameters of 2-phenyl-2-imidazoline obtained by B3LYP/ 6-311+G** density functional calculations.

\begin{tabular}{|c|c|c|c|c|c|}
\hline \multicolumn{3}{|c|}{ Bond length $\left(A^{0}\right)^{a}$} & \multicolumn{3}{|c|}{ Bond angle $\left({ }^{0}\right)^{\mathrm{a}}$} \\
\hline \multicolumn{2}{|c|}{ B3LYP/6-31G(d,p) } & \multirow{2}{*}{$\begin{array}{c}\text { Exp } \\
1.498^{\mathrm{b}}\end{array}$} & \multicolumn{2}{|c|}{ B3LYP/6-31G(d,p) } & \multirow{2}{*}{$\begin{array}{c}\text { Exp } \\
101.3^{\mathrm{b}}\end{array}$} \\
\hline C1-N11 & 1.445 & & N11-C1-C2 & 101.2 & \\
\hline $\mathrm{C} 1-\mathrm{C} 2$ & 1.528 & $1.550^{\mathrm{b}}$ & $\mathrm{C} 1-\mathrm{C} 2-\mathrm{N} 10$ & 106.2 & $102.1^{\mathrm{b}}$ \\
\hline $\mathrm{C} 2-\mathrm{N} 10$ & 1.488 & $1.488^{\mathrm{b}}$ & $\mathrm{C} 1-\mathrm{C} 2-\mathrm{H} 14$ & 113.3 & - \\
\hline C3-N10 & 1.311 & $1.341^{\mathrm{b}}$ & N10-C3-N11 & 115.9 & $108.8^{\mathrm{b}}$ \\
\hline C3-N11 & 1.377 & $1.346^{\mathrm{b}}$ & C3-C4-C5 & 119.5 & $120.7^{\mathrm{b}}$ \\
\hline $\mathrm{C} 3-\mathrm{C} 4$ & 1.485 & $1.458^{\mathrm{b}}$ & C3-C4-C7 & 120.7 & $119.9^{\mathrm{b}}$ \\
\hline C4-C5 & 1.399 & $1.381^{\mathrm{b}}$ & $\mathrm{C} 5-\mathrm{C} 4-\mathrm{C} 7$ & 119.6 & $119.4^{\mathrm{b}}$ \\
\hline $\mathrm{C} 4-\mathrm{C} 7$ & 1.399 & $1.391^{\mathrm{b}}$ & $\mathrm{C} 2-\mathrm{C} 1-\mathrm{H} 20$ & 112.5 & - \\
\hline C7-H18 & 1.085 & $0.930^{\mathrm{b}}$ & C9-C8-C6 & 120.0 & $121.5^{\mathrm{b}}$ \\
\hline C7-C9 & 1.397 & $1.352^{\mathrm{b}}$ & C8-C6-C5 & 120.0 & $119.3^{\mathrm{b}}$ \\
\hline $\mathrm{C} 9-\mathrm{C} 8$ & 1.394 & $1.394^{\mathrm{b}}$ & C6-C5-C4 & 120.1 & $119.3^{\mathrm{b}}$ \\
\hline $\mathrm{C} 8-\mathrm{C} 6$ & 1.394 & $1.369^{\mathrm{b}}$ & C4-C7-H18 & 119.6 & $119.4^{\mathrm{b}}$ \\
\hline C6-C5 & 1.396 & $1.394^{\mathrm{b}}$ & C9-C7-H18 & 119.6 & $119.4^{\mathrm{b}}$ \\
\hline C9-H19 & 1.087 & $0.930^{\mathrm{b}}$ & C7-C9-H19 & 119.9 & $120.4^{\mathrm{b}}$ \\
\hline C8-H17 & 1.086 & $0.930^{\mathrm{b}}$ & C8-C9-H19 & 119.9 & $120.4^{\mathrm{b}}$ \\
\hline C6-H16 & 1.086 & $0.930^{\mathrm{b}}$ & C9-C8-H17 & 120.0 & $119.2^{\mathrm{b}}$ \\
\hline \multirow[t]{5}{*}{ C5-H15 } & 1.087 & $0.930^{\mathrm{b}}$ & C6-C8-H17 & 119.9 & $119.2^{\mathrm{b}}$ \\
\hline & & & C8-C6-H16 & 120.1 & $120.4^{\mathrm{b}}$ \\
\hline & & & C5-C6-H16 & 119.8 & $120.4^{\mathrm{b}}$ \\
\hline & & & N11-C3-C4 & 122.5 & $125.6^{\mathrm{b}}$ \\
\hline & & & $\mathrm{N} 10-\mathrm{C} 3-\mathrm{C} 4$ & 121.5 & $125.5^{\mathrm{b}}$ \\
\hline
\end{tabular}




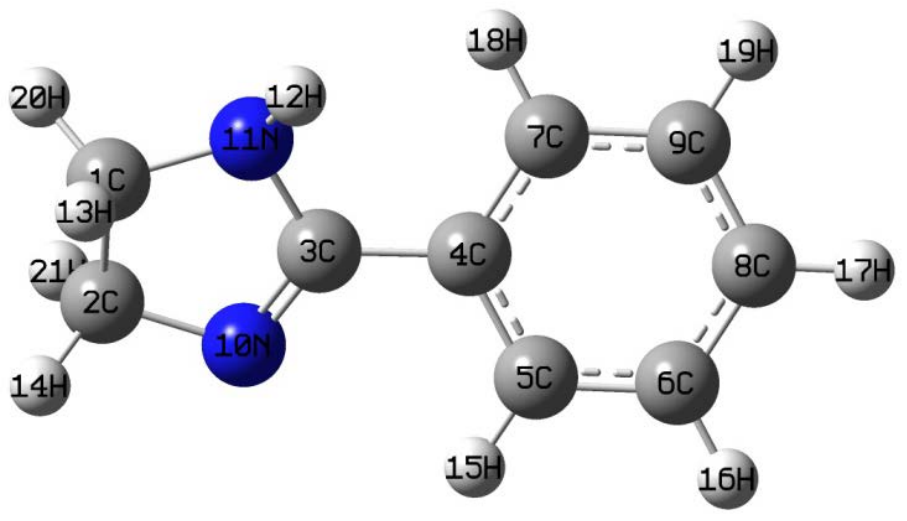

Figure 1. Molecular structure of 2-phenyl-2-imidazoline along with numbering of atom.

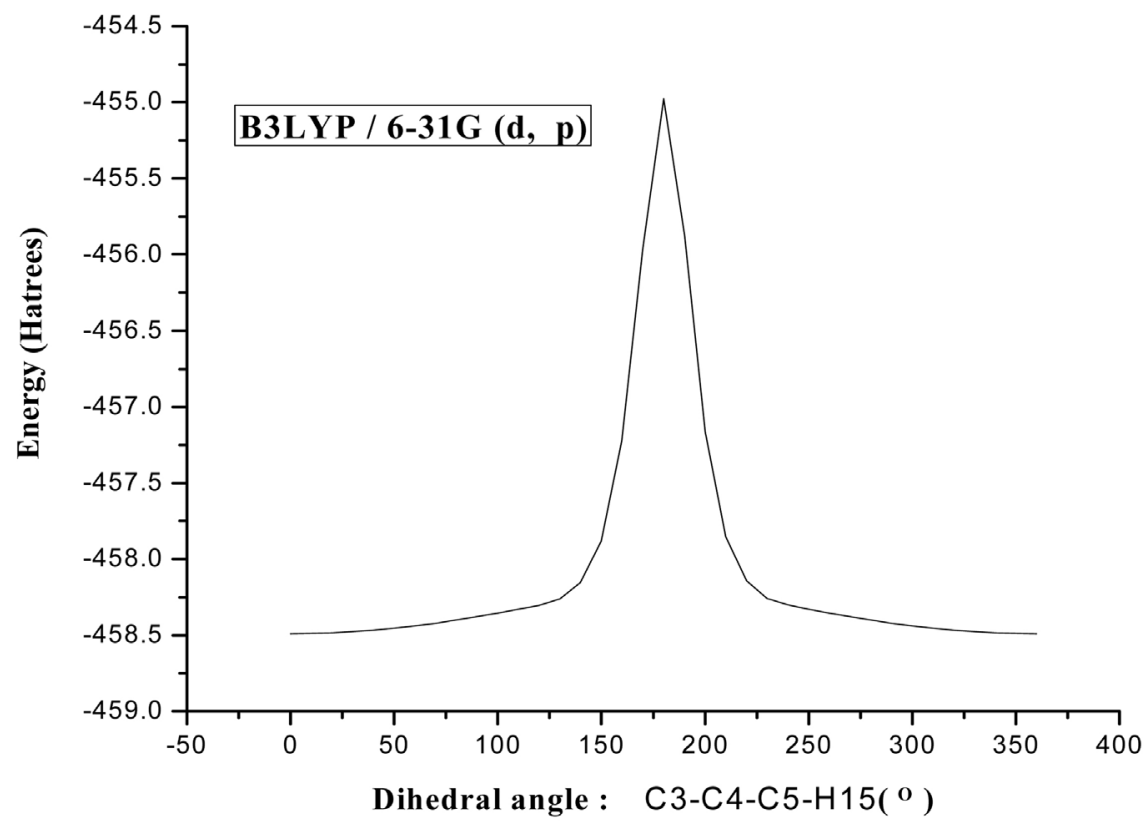

Figure 2. Potential energy surface scan for dihedral angle C3-C4-C5-H15 of 2-phenyl-2-imidazoline.

be because of the intermolecular interactions during the crystalline state. Further, it is noticed that the differences are more for $\mathrm{C}-\mathrm{H}$ bonds and less for the $\mathrm{C}-\mathrm{C}$ and $\mathrm{C}-\mathrm{N}$ bonds. The differences are of the order of $0 \mathrm{~A}^{\circ} \pm 0.156 \mathrm{~A}^{\circ}$ for the Bond length when compared with the investigational data [20]. It is observed that the theoretical values are somewhat more than the experimental values for bond lengths. This difference may be because of the cause that the theoretical computations were meant by the molecule in the gaseous phase isolated compound and the experimental outcomes were meant by the molecule in the solid phase. Additionally it is observed that the bond angles are in reasonable agreement with the experimental data of the corresponding observed theoretical data. In spite of the above differences the calculated geometries are used as a foundation to calculate the properties of the molecule such as vibrational frequencies, 
polarizability and other properties which are discussed in the following sessions.

Both infrared and Raman spectra. A complete description of fundamental vibrational frequencies of the title molecule was given by Normal coordinate analysis. For this purpose a set of internal coordinates are defined in Supplementary Material 1. The local and non redundant symmetry coordinates are defined from these predefined internal coordinates following the recommendations of Fogarasi and Pulay [21] [22]. The detailed description of symmetry coordinates and their respective scale factors are reported in Table 2. The simulated and experimental FTIR and FT Raman spectra are shown in Figure 3 and Figure 4 respectively.

The title molecule belongs to $C_{1}$ symmetry. The 57 modes of vibrations of the molecule are distributed as 39 in plane and 18 out of plane modes. All the vibrational modes are active in:

\subsection{Vibrational Analysis}

\subsubsection{C-H Vibrations}

Aromatic compounds have one or more sharp peaks of weak or medium intensity between $3100-3000 \mathrm{~cm}^{-1}$ [23]. The expected five C-H stretching vibrations of the title compound correspond to mode nos. 13 - 17 of the benzene ring. A weak band observed at $3065 \mathrm{~cm}^{-1}$ FT-Raman spectrum belongs to the cluster consisting of the five $\mathrm{C}-\mathrm{H}$ stretching vibrational bands. The vibrations are shown in PED matrix with percentage about $99 \%$.

The $\mathrm{C}-\mathrm{H}$ in-plane bending vibrations of benzene and its derivatives are observed in the region $1300-1000 \mathrm{~cm}^{-1}$ [24]. In this region the bands are sharp, but are of weak to medium intensity. The calculated frequency $1122 \mathrm{~cm}^{-1}$ is assigned to $\mathrm{C}-\mathrm{H}$ in-plane bending vibration and this is in good agreement with the recorded FT-IR spectrum at $1122 \mathrm{~cm}^{-1}$. The $\mathrm{C}-\mathrm{H}$ out of plane bending vibrations are observed at frequencies at $986 \mathrm{~cm}^{-1}$ and $915 \mathrm{~cm}^{-1}$ which are coinciding very well with the experimental FT-IR Spectrum at $983 \mathrm{~cm}^{-1}$ and FT-Raman Spectrum at $912 \mathrm{~cm}^{-1}$ respectively. The Maximum PED distribution is $68 \%$ for $\mathrm{C}-\mathrm{H}$ out of plane bending vibrations. The Remaining calculated $\mathrm{C}-\mathrm{H}$ out of plane bending vibrations is observed at frequencies $955 \mathrm{~cm}^{-1}, 840 \mathrm{~cm}^{-1}$ and $720 \mathrm{~cm}^{-1}$ with a PED Distribution of $41 \%, 39 \%$, and $55 \%$ respectively.

\subsubsection{Methylene Group Vibrations}

The asymmetric $\mathrm{CH}_{2}$ stretching vibration is generally observed in the region $3000-2900 \mathrm{~cm}^{-1}$, while the $\mathrm{CH}_{2}$ symmetric stretch will appear between 2900 and $2800 \mathrm{~cm}^{-1}$ [25] [26] [27]. The $\mathrm{CH}_{2}$ asymmetric stretching vibrations are assigned to calculated frequencies at 2955 and $2942 \mathrm{~cm}^{-1}$ and a week peak is observed at frequency $2946 \mathrm{~cm}^{-1}$ FT-IR spectrum. The symmetric $\mathrm{CH}_{2}$ stretching vibrations are assigned to scaled vibrational frequencies at 2890 and $2874 \mathrm{~cm}^{-1}$ respectively. These modes are not observed experimentally both in FT-IR and FT-Raman spectrum. The scissoring vibrations are expected in the region $1455-1380 \mathrm{~cm}^{-1}$ 
Table 2. Definition of local-symmetry coordinates and the values of corresponding scale factors Used to correct the B3LYP/6-311++G** (refined) force field of 2-phenyl-2-imidazoline.

\begin{tabular}{|c|c|c|c|}
\hline No.(i) & Symbol $^{\mathrm{a}}$ & Definition $^{\mathrm{b}}$ & Scale factors \\
\hline \multicolumn{4}{|c|}{ Stretching } \\
\hline $1-8$ & $v(\mathrm{C}-\mathrm{C})$ & R1, R2, R3, R4, R5, R6, R7, R8 & 0.94293 \\
\hline $9-12$ & $v(\mathrm{C}-\mathrm{N})$ & $\mathrm{r} 9, \mathrm{r} 10, \mathrm{r} 11, \mathrm{r} 12$ & 0.9461 \\
\hline $13-17$ & $v(\mathrm{C}-\mathrm{H})$ & P13, P14, P15, P16, P17 & 0.9225 \\
\hline $18-19$ & $v(\mathrm{CH} 2 \mathrm{ss})$ & $(\mathrm{P} 18+\mathrm{P} 19) / / 2,(\mathrm{P} 20+\mathrm{P} 21) / / 2$ & 0.9192 \\
\hline $20-21$ & $v$ (CH2ass) & $(\mathrm{P} 18-\mathrm{P} 19) / / 2,(\mathrm{P} 20-\mathrm{P} 21) / / 2$ & 0.9192 \\
\hline 22 & $v(\mathrm{~N}-\mathrm{H})$ & Q22 & 0.91287 \\
\hline \multicolumn{4}{|c|}{ In-Plane bending } \\
\hline 23 & $\beta \mathrm{R} 1$ tri & $\left(\beta_{23}-\beta_{24}+\beta_{25}-\beta_{26}+\beta_{27}-\beta_{28}\right) / \sqrt{6}$ & 0.96557 \\
\hline 24 & $\beta \mathrm{R} 1 \mathrm{sym}$ & $\left(-\beta_{23}-\beta_{24}+2 \beta_{25}-\beta_{26}-\beta_{27}+2 \beta_{28}\right) / \sqrt{12}$ & 0.96557 \\
\hline 25 & $\beta$ R1asy & $\left(\beta_{23}-\beta_{24}+\beta_{27}-\beta_{28}\right) / 2$ & 0.96557 \\
\hline 26 & R2bend1 & $\beta_{29}+a\left(\beta_{30}+\beta_{33}\right)+b\left(\beta_{31}-\beta_{32}\right)$ & 0.956 \\
\hline 27 & R2bend2 & $(a-b)\left(\beta_{30}-\beta_{33}\right)+(1-a)\left(\beta_{31}+\beta_{32}\right)$ & 0.956 \\
\hline $28-33$ & $\mathrm{bCH}$ & $\begin{array}{l}\left(\theta_{34}-\theta_{35}\right) / \sqrt{2},\left(\theta_{36}-\theta_{37}\right) / \sqrt{2},\left(\theta_{38}-\theta_{39}\right) / \sqrt{2} \\
\left(\theta_{40}-\theta_{41}\right) / \sqrt{2},\left(\theta_{42}-\theta_{43}\right) / \sqrt{2},\left(\theta_{44}-\theta_{45}\right) / \sqrt{2}\end{array}$ & 0.9466 \\
\hline 34 & $\mathrm{bNH}$ & $\left(\theta_{46}-\theta_{47}\right) / \sqrt{2}$ & 0.9466 \\
\hline $35-36$ & bCC & $\left(\theta_{48}-\theta_{49}\right) / \sqrt{2},\left(\theta_{50}-\theta_{51}\right) / \sqrt{2}$ & 0.9265 \\
\hline $37-38$ & $\mathrm{bCH} 2 \mathrm{sc}$ & $\alpha_{52}+\alpha_{53}+\alpha_{54}+\alpha_{55}, \alpha_{56}+\alpha_{57}+\alpha_{58}+\alpha_{59}$ & 0.95715 \\
\hline $39-40$ & bCH2Roc & $\alpha_{52}+\alpha_{53}-\alpha_{54}-\alpha_{55}, \alpha_{56}+\alpha_{57}-\alpha_{58}-\alpha_{59}$ & 0.95715 \\
\hline $41-42$ & bCH2Wag & $\alpha_{52}-\alpha_{53}-\alpha_{54}+\alpha_{55}, \alpha_{56}-\alpha_{57}-\alpha_{58}+\alpha_{59}$ & 0.95715 \\
\hline \multicolumn{4}{|c|}{ Out of plane bending } \\
\hline $43-47$ & $\omega \mathrm{C}-\mathrm{H}$ & $\omega_{60}, \omega_{61}, \omega_{62}, \omega_{63}, \omega_{64}$ & 0.9654 \\
\hline 48 & $\omega \mathrm{N}-\mathrm{H}$ & $\omega_{65}$ & 0.9654 \\
\hline $49-50$ & $\omega \mathrm{C}-\mathrm{C}$ & $\omega_{66}, \omega_{67}$ & 0.98700 \\
\hline \multicolumn{4}{|c|}{ Torsion } \\
\hline 51 & $\tau \mathrm{R} 1 \mathrm{tri}$ & $\left(\tau_{68}-\tau_{69}+\tau_{70}-\tau_{71}+\tau_{72}-\tau_{73}\right) / \sqrt{6}$ & 0.98867 \\
\hline 52 & $\tau$ R1asy & $\left(\tau_{68}-\tau_{70}+\tau_{71}-\tau_{73}\right) / 2$ & 0.98867 \\
\hline 53 & $\tau \mathrm{R} 1$ sym & $\left(-\tau_{68}-2 \tau_{69}-\tau_{70}-\tau_{71}+2 \tau_{72}-\tau_{73}\right) / \sqrt{12}$ & 0.98867 \\
\hline 54 & R2torsion1 & $\tau_{76}+b\left(\tau_{74}+\tau_{78}\right)+a\left(\tau_{75}+\tau_{77}\right)$ & 0.9966 \\
\hline 55 & R2torsion2 & $(a-b)\left(\tau_{75}-\tau_{77}\right)+(1-a)\left(\tau_{74}-\tau_{78}\right)$ & 0.9966 \\
\hline 56 & $\tau \mathrm{CH}_{2}$ & $\tau_{79}+\tau_{80}$ & 0.980 \\
\hline 57 & $\tau \mathrm{CCCC}$ & $\tau_{81}+\tau_{82}$ & 1.2000 \\
\hline
\end{tabular}

$a=\cos 144^{\circ}$, and $b=\cos 72^{\circ}$.

and consist of medium intense bands [25] [28] [29]. In Table 3, $\mathrm{CH}_{2}$ scissoring vibration has been assigned to scaled frequency at $1447 \mathrm{~cm}^{-1}$ and the corresponding frequency is observed at $1454 \mathrm{~cm}^{-1}$ in FT-Raman spectrum. The $\mathrm{CH}_{2}$ twisting vibration and is predicted theoretically at $1004 \mathrm{~cm}^{-1}$ and it is observed at 


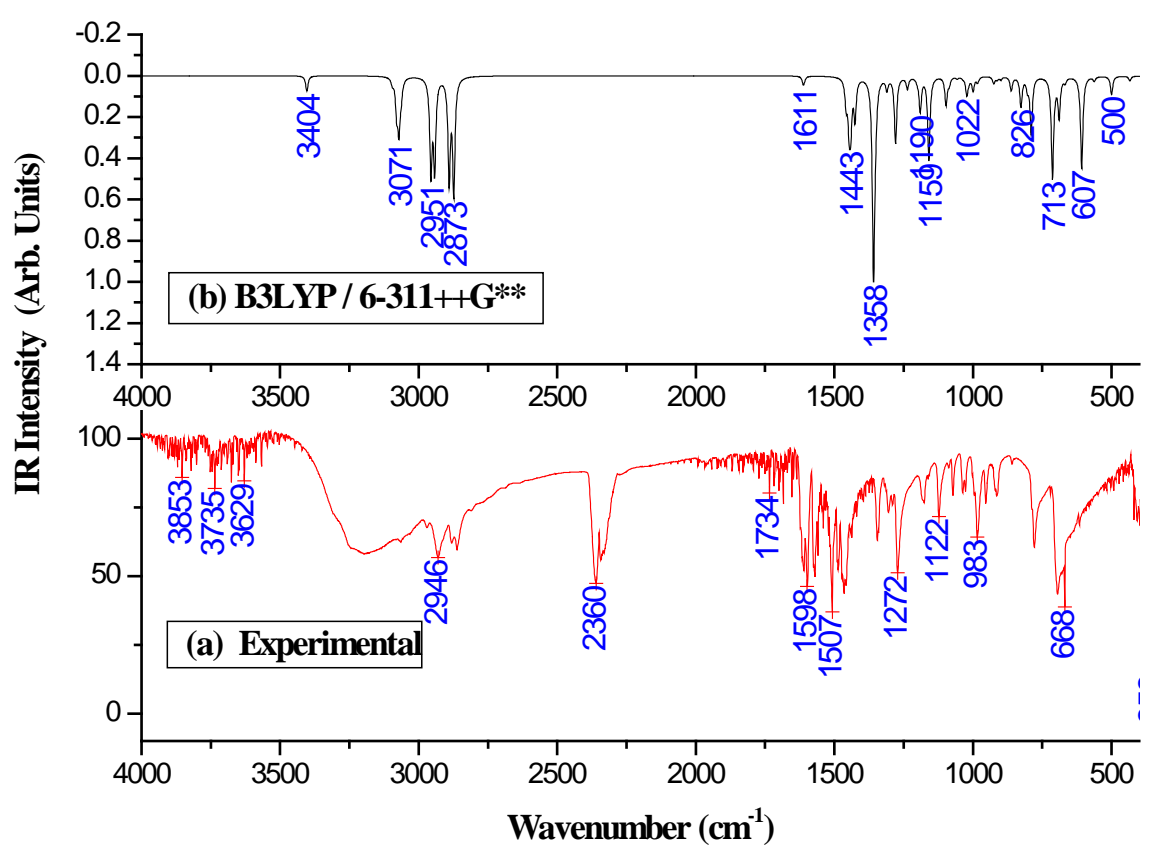

Figure 3. (a) Experimental, (b) Simulated FT-IR spectra of 2-phenyl-2-imidazoline.

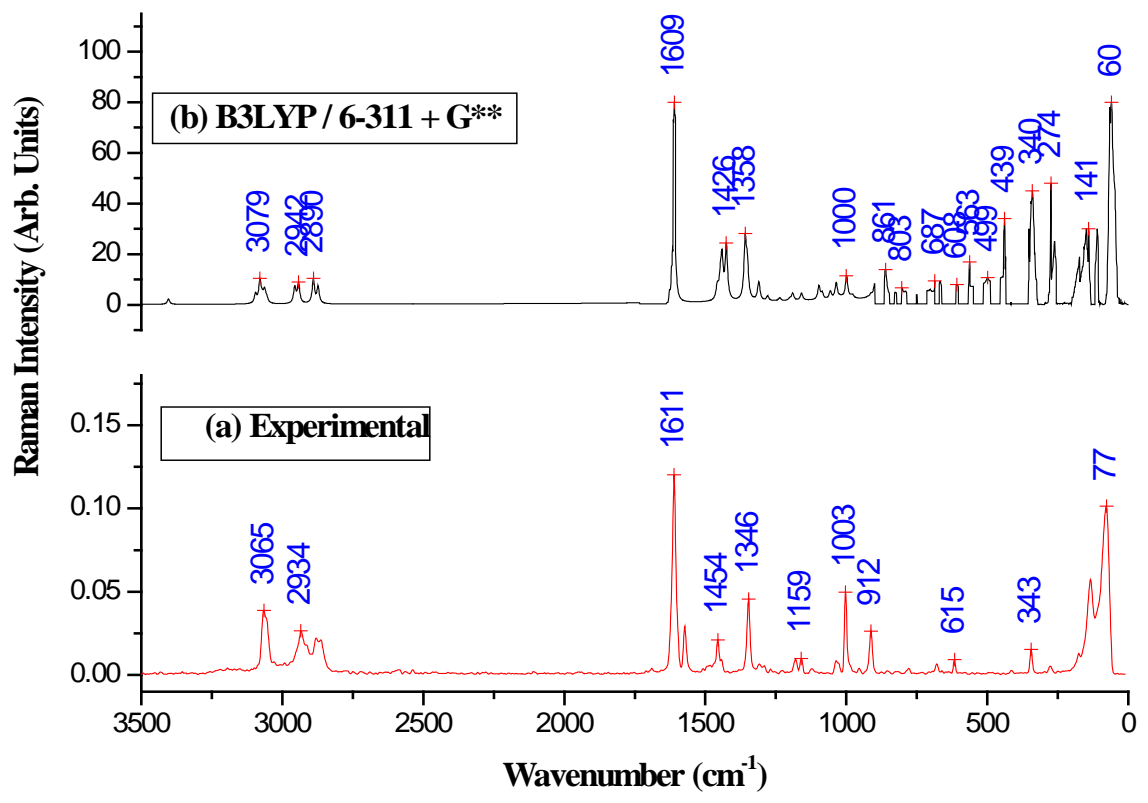

Figure 4. (a) Experimental and (b) simulated FT-Raman spectra of 2-phenyl-2-imidazoline.

$1003 \mathrm{~cm}^{-1}$ in FT-Raman spectrum. This shows an excellent agreement with each other. The $\mathrm{CH}_{2}$ wagging vibrations are observed theoretically with less PED distribution.

\subsubsection{C-C Vibrations}

The bands between 1400 and $1650 \mathrm{~cm}^{-1}$ in benzene derivatives are due to C-C stretching vibrations [30] [31]. The strong FT-RAMAN band at $1611 \mathrm{~cm}^{-1}$ and a weak FT-IR band at $1598 \mathrm{~cm}^{-1}$ are assigned to C-C stretching vibration and are 
Table 3. Detailed assignments of fundamental vibrations of 2-phenyl-2-imidazoline by normal mode analysis based on SQM force field calculations using B3LYP/6-311++G**.

\begin{tabular}{|c|c|c|c|c|c|c|}
\hline \multirow[b]{2}{*}{ s.no } & \multicolumn{2}{|c|}{ Experimental $\left(\mathrm{cm}^{-1}\right)$} & \multirow{2}{*}{$\begin{array}{c}\text { Scaled } \\
\text { frequencies } \\
\left(\mathrm{cm}^{-1}\right)\end{array}$} & \multicolumn{2}{|c|}{ Intensity } & \multirow{2}{*}{ Characterization of normal modes with PED $(\%)^{\mathrm{a}, \mathrm{d}}$} \\
\hline & FT-IR & FT-Raman & & $\mathrm{I}_{\mathrm{IR}}^{\mathrm{b}}$ & $I_{R A}{ }^{c}$ & \\
\hline 1. & $3404 \mathrm{vw}$ & & 3398 & 0.033 & 1.12 & $\mathrm{vNH}(89)$ \\
\hline 2 & & & 3096 & 0.055 & 4.67 & vCH(99) \\
\hline 3 & & & 3081 & 0.205 & 9.44 & $\mathrm{vCH}(99)$ \\
\hline 4 & & & 3072 & 0.309 & 6.51 & $\mathrm{vCH}(99)$ \\
\hline 5 & & $3065 w$ & 3063 & 0.158 & 7.04 & $\mathrm{vCH}(99)$ \\
\hline 6 & & & 3054 & 0.049 & 3.65 & vCH(99) \\
\hline 7. & & & 2955 & 0.511 & 7.62 & $\mathrm{vCH} 2 \mathrm{as}(78), \mathrm{vCH} 2 \mathrm{ss}(18)$ \\
\hline 8 & $2946 w$ & & 2942 & 0.496 & 9.02 & $\mathrm{vCH} 2 \mathrm{as}(65), \mathrm{vCH} 2 \mathrm{ss}(30)$ \\
\hline 9 & & & 2890 & 0.546 & 10.4 & $\mathrm{vCH} 2 \mathrm{ss}(75), \mathrm{vCH} 2 \mathrm{as}(21)$ \\
\hline 10 & & & 2874 & 0.59 & 7.46 & vCH2ss(59), vCH2as(26), bR2sym(10) \\
\hline 11 & & & 2192 & 0.001 & 0.45 & $\operatorname{gCC}(61), \operatorname{tCCCC}(38)$ \\
\hline 12 & $1598 w$ & $1611 \mathrm{~s}$ & 1605 & 0.021 & 20.8 & vCC(69), bCH(17), bR1sym(10) \\
\hline 13 & & & 1582 & 0.003 & 2.02 & $\mathrm{vCC}(50), \mathrm{vCN}(15), \mathrm{bR} 2 \operatorname{sym}(14), \mathrm{bCH}(10)$ \\
\hline 14 & & & 1473 & 0.029 & 2.43 & bR2sym(25), vCC(22), bCH(20), vCN(16), gCC(10) \\
\hline 15 & & $1454 w$ & 1447 & 0.339 & 16.4 & bCH2sc(29), bR2sym(25), gCC(16), vCN(14), tCCCC(10) \\
\hline 16 & & & 1446 & 0.353 & 17.6 & vCN(51), bR2sym(26), bCH2sc(15) \\
\hline 17 & & & 1428 & 0.208 & 20.9 & $\mathrm{vCN}(39), \mathrm{bR} 2 \operatorname{sym}(20), \mathrm{bCH}(15), \mathrm{vCC}(12)$ \\
\hline 18 & & & 1382 & 0.053 & 2.95 & bR2sym(35), vCC(16), vCN(12), gC(11) \\
\hline 19 & & $1346 s$ & 1355 & 0.720 & 24.2 & vCN(49), bR2sym(37) \\
\hline 20 & & & 1308 & 0.069 & 7.83 & $\mathrm{vCN}(38), \mathrm{vCC}(27), \mathrm{bR} 2 \operatorname{sym}(18)$ \\
\hline 21 & $1272 s$ & & 1279 & 0.331 & 3.61 & bR2sym(31), vCC(22), vCN(14), bCH2wa(10) \\
\hline 22 & & & 1263 & 0.037 & 1.82 & bR2sym(31), vCC(22), vCN(14), bCH2wa(10) \\
\hline 23 & & & 1230 & 0.033 & 2.12 & bNH(28)vCN(27), bR2sym(26) \\
\hline 24 & & & 1194 & 0.121 & 3.74 & $\mathrm{vCN}(51), \mathrm{gCC}(13), \mathrm{bCH} 2 \mathrm{wa}(10)$ \\
\hline 25 & & $1159 w$ & 1168 & 0.107 & 2.53 & $\mathrm{vCN}(49), \mathrm{tCH} 2(22), \mathrm{bCH} 2 \mathrm{wa}(12)$ \\
\hline 26 & & & 1142 & 0.038 & 2.09 & $\mathrm{bCH}(66), \mathrm{vCC}(14)$ \\
\hline 27 & $1122 \mathrm{w}$ & & 1122 & 0.016 & 2.13 & bCH(86), vCC(11) \\
\hline 28 & & & 1092 & 0.085 & 5.58 & bCH(28), bR2sym(21), bCH2wa(17), vCC(15) \\
\hline 29 & & & 1065 & 0.012 & 3.27 & $\operatorname{vCN}(53), \mathrm{vCC}(22)$ \\
\hline 30 & & & 1052 & 0.013 & 4.38 & vCN(35), vCC(32), bCH(18) \\
\hline 31 & & & 1017 & 0.065 & 4 & vCC(46), bCH(17), bR1tri(17), vCN(14) \\
\hline 32 & & $1003 \mathrm{~s}$ & 1004 & 0.049 & 7.41 & bCH2Ro(48), tR2asy(19), tCCCC(13) \\
\hline 33 & & & 997 & 0.067 & 10.1 & bR1tri (49), vCC(42) \\
\hline 34 & $983 \mathrm{~s}$ & & 986 & 0.035 & 4.24 & $\mathrm{gCH}(68), \mathrm{tR} 1 \operatorname{tri}(21)$ \\
\hline 35 & & & 955 & 0.005 & 2.71 & $\mathrm{gCH}(41), \mathrm{tCCCC}(30)$ \\
\hline 36 & & $912 \mathrm{~s}$ & 915 & 0.023 & 4.33 & $\mathrm{gCH}(65)$ \\
\hline 37 & & & 910 & 0.021 & 4.79 & $\mathrm{vCC}(60), \mathrm{vCN}(27)$ \\
\hline
\end{tabular}




\section{Continued}

\begin{tabular}{|c|c|c|c|c|c|c|}
\hline 38 & & & 840 & 0.027 & 0.37 & $\mathrm{gCH}(39), \mathrm{bR} 2 \operatorname{sym}(28), \mathrm{tCCCC}(13), \mathrm{vCC}(13)$ \\
\hline 39 & & & 839 & 0.0294 & 0.37 & bR2sym(52), vCC(20), vCN(19) \\
\hline 40 & & & 805 & 0.0837 & 0.37 & $\mathrm{vCN}(44), \mathrm{bR} 2 \operatorname{sym}(22), \mathrm{tR} 1 \operatorname{tri}(17)$ \\
\hline 41 & & & 790 & 0.289 & 5.4 & $\operatorname{vCN}(67)$, bR2sym(10) \\
\hline 42 & & & 769 & 0.0234 & 1.4 & $\mathrm{vCN}(44), \mathrm{gCC}(24), \mathrm{bR} 2 \operatorname{sym}(16), \mathrm{tCCCC}(13)$ \\
\hline 43 & & & 720 & 0.172 & 3.6 & $\operatorname{gCH}(55), \mathrm{vCN}(23)$ \\
\hline 44 & & & 694 & 0.135 & 5.29 & bR2sym(49), vCN(33) \\
\hline 45 & $668 w$ & & 660 & 0.0228 & 3.8 & bR2sym(61), vCN(18) \\
\hline 46 & & $615 \mathrm{w}$ & 621 & 0.0575 & 4.2 & bR1asy(69) \\
\hline 47 & & & 592 & 0.0459 & 1.65 & bR2sym(42), vCN(35), gCC(13) \\
\hline 48 & & & 522 & 0.00727 & 4 & bR2sym(44), vCN(39) \\
\hline 49 & & & 477 & 0.00617 & 0.52 & bR2sym(55), vCN(41) \\
\hline 50 & & & 450 & 0.00435 & 10.8 & $\operatorname{tCCCC}(94)$ \\
\hline 51 & & $343 w$ & 347 & 0.0162 & 32 & bR2sym(68), vCN(31) \\
\hline 52 & & & 310 & 0.0102 & 0.61 & bR2sym(42), vCN(41) \\
\hline 53 & & & 276 & 0.367 & 35 & bR2sym(34), tCCCC(28), tR2asy(18), tR2sym(12) \\
\hline 54 & & & 169 & 0.0199 & 11 & tCCCC(67), tCH2(14), tR2asy(11) \\
\hline 55 & & & 142 & 0.0416 & 17 & bR2sym(58), vCN(19), tCCCC(12) \\
\hline 56 & & & 115 & 0.0344 & 18 & $\operatorname{tCCCC}(40)$, bR2sym(36) \\
\hline 57 & & $77 \mathrm{~s}$ & 77 & 0.00256 & 4 & $\operatorname{tCCCC}(100)$ \\
\hline
\end{tabular}

a Abbreviations: v, stretching; b, in plane bending; g, out of plane bending; t, torsion; ss, symmetrical stretching; as, asymmetrical stretching; tri, trigonal deformation; sym, symmetrical deformation; asy, asymmetric deformation, vs, very strong; s, strong; m, medium; w, weak; vw, very weak; ${ }^{\mathrm{b}}$ Relative absorption intensities normalized with highest peak absorption equal to 1 . ${ }^{\mathrm{c}}$ Relative Raman intensities calculated by Equation (1) and normalized to 100 . ${ }^{\mathrm{d}}$ Only PED contributions $\geq 10 \%$ are listed.

theoretically observed at frequency $1605 \mathrm{~cm}^{-1}$. The remaining C-C stretching vibrations are observed theoretically at $1582 \mathrm{~cm}^{-1}$ and $1012 \mathrm{~cm}^{-1}$ and some of the frequencies are having less PED distribution. The C-C out of plane vibration predicted theoretically at $2192 \mathrm{~cm}^{-1}$ and no Experimental bands were observed.

\subsubsection{N-H Vibrations}

Hetero cyclic compound having an $\mathrm{N}-\mathrm{H}$ group shows its stretching absorption in the region $3500-3200 \mathrm{~cm}^{-1}$ [32]. The peak of absorption in this region depends on the degree of hydrogen bonding along with the physical state of the sample or the Polarity of the solvent. A very weak band observed at $3404 \mathrm{~cm}^{-1}$ in FT-IR spectrum and the scaled frequency at $3398 \mathrm{~cm}^{-1}$ are assigned to $\mathrm{N}-\mathrm{H}$ stretching vibrations. Further, the $\mathrm{N}-\mathrm{H}$ in plane bending modes are scaled at $1230 \mathrm{~cm}^{-1}$.

\subsubsection{Ring Vibrations}

The ring stretching vibrations have great significance during the Infrared spectrum of benzene derivatives since ring stretching vibrations include high characteristic modes of the aromatic rings. As a result of the substitution to the 
aromatic ring of benzene derivatives several ring vibrations be affected. The skeletal vibrations are due to the coupled vibrations in the six ring carbon atoms. The semi circle stretching vibrations such as ring $\mathrm{C}=\mathrm{C}$ and $\mathrm{C}-\mathrm{C}$ vibrations take place in the region $1400-1625 \mathrm{~cm}^{-1}$. Due to the electro negativity of nitrogen atom there are small changes in frequencies are observed for these modes. Apart from ring $\mathrm{C}-\mathrm{C}$ stretching modes, trigonal deformation for related benzene ring, symmetric and asymmetric deformation modes for both the rings are assigned as listed in Table 3.

\section{NBO Analysis}

$\mathrm{NBO}$ analysis for the title compound was carried out at the $6-311++\mathrm{G}^{\star *}$ basis set to calculate delocalization of the electron density within the molecule. NBO calculations are used to understand the interactions between filled and virtual orbitals of one subsystem with another subsystem. These interactions possibly will enhance the analysis of intra- and inter molecular interactions. The hyperconjugative interaction energy is deduced from the second-order perturbation approach [33] [34]. The intramolecular hyper interactions are formed by the orbital overlap between bonding $(\mathrm{C}-\mathrm{C}),(\mathrm{C}-\mathrm{N})$ and $(\mathrm{C}-\mathrm{C}),(\mathrm{C}-\mathrm{N})$ anti bonding orbital which effects intramolecular charge transfer (ICT) causing stabilization of the system.

These interactions are observed as raise in electron density (ED) in C-C, C-N antibonding orbital to weaken the particular bonds. As listed in Table 4, the hyper conjugative interaction between $\mathrm{C} 2-\mathrm{N} 10$ and $\mathrm{C} 3-\mathrm{C} 4$ leads to stabilization energy of $8.65 \mathrm{KJ} / \mathrm{mol}$. The anti bonding orbital of $\sigma^{*} \mathrm{C} 4-\mathrm{C} 7$ and $\mathrm{C} 8-\mathrm{C} 9$ in conjugation with bonding orbital C5-C6 show the strong delocalization of 19.94 and $21.05 \mathrm{KJ} / \mathrm{mol}$ respectively. Similarly the anti bonding orbital of $\sigma^{*} \mathrm{C} 4-\mathrm{C} 7$ and C5-C6 in conjugation with bonding orbital C8-C9 leads to the strong delocalization of 20.44 and $18.58 \mathrm{KJ} / \mathrm{mol}$. respectively. Further, the $\sigma^{\star}(\mathrm{C} 4-\mathrm{C} 7)$ of the NBO in conjugation with $\sigma^{\star}(\mathrm{C} 3-\mathrm{N} 10)$ resulting a strong stabilization energy of 118.65 $\mathrm{KJ} / \mathrm{mol}$. These data signifies the strong hyperconjugative intramolecular interactions in the molecule and explains the stability of the molecule.

\section{NLO Properties}

Non linear optical (NLO) materials play a vital role in optical switching devices and industrial applications. The first hyperpolarizability $\beta$, dipole moment $\mu$ as well as polarizability $\alpha$ are computed using HF/6-31G (d, p) basis set on the basis of the finite-field approach. The complete equations for calculating the magnitude of total static dipole moment $\mu$, the mean polarizability $\alpha_{0}$, the anisotropy of the polarizability $\Delta \alpha$ and the mean first hyperpolarizability $\beta_{0}$, using the $x, y, z$ components from Gaussian 03W output are as follows

$$
\begin{gathered}
\mu=\mu_{x}^{2}+\mu_{y}^{2}+\mu_{z}^{2} \\
\alpha_{o}=\frac{\alpha_{x x}+\alpha_{y y}+\alpha_{z z}}{3}
\end{gathered}
$$


Table 4. Second order perturbation theory analysis of fock matrix in NBO basis for 2-phenyl-2-imidazoline.

\begin{tabular}{|c|c|c|c|c|c|c|c|c|}
\hline Donor(i) & Type & $\mathrm{E}_{\mathrm{d}} / \mathrm{e}$ & Acceptor(j) & Type & $\mathrm{E}_{\mathrm{d}} / \mathrm{e}$ & $\begin{array}{c}\mathrm{E}^{(2) \mathrm{a}} \\
\left(\mathrm{kJ} \cdot \mathrm{mol}^{-1}\right)\end{array}$ & $E(i)-E(j)^{b}(a \cdot u)$ & $f(I, j)^{c}(a . u)$ \\
\hline $\mathrm{C} 1-\mathrm{C} 2$ & $\sigma$ & 1.98898 & N11-H12 & $\sigma^{*}$ & 0.01847 & 2.68 & 1.05 & 0.047 \\
\hline C1-N11 & $\sigma$ & 1.98586 & $\mathrm{C} 3-\mathrm{C} 4$ & $\sigma^{*}$ & 0.03966 & 4.34 & 1.18 & 0.064 \\
\hline $\mathrm{C} 1-\mathrm{H} 13$ & $\sigma$ & 1.98923 & $\mathrm{C} 2-\mathrm{H} 21$ & $\sigma^{*}$ & 0.02002 & 1.60 & 0.97 & 0.035 \\
\hline $\mathrm{C} 1-\mathrm{H} 20$ & $\sigma$ & 1.98675 & C3-N11 & $\sigma^{*}$ & 0.04973 & 1.59 & 0.95 & 0.035 \\
\hline $\mathrm{C} 2-\mathrm{N} 10$ & $\sigma$ & 1.98133 & $\mathrm{C} 3-\mathrm{C} 4$ & $\sigma^{*}$ & 0.03966 & 8.65 & 1.14 & 0.089 \\
\hline C2-H14 & $\sigma$ & 1.98369 & $\mathrm{C} 3-\mathrm{N} 10$ & $\sigma^{*}$ & 0.23563 & 1.74 & 0.55 & 0.029 \\
\hline $\mathrm{C} 2-\mathrm{H} 21$ & $\sigma$ & 1.97896 & C3-N10 & $\sigma^{*}$ & 0.23563 & 2.00 & 0.55 & 0.031 \\
\hline \multirow[t]{4}{*}{ C3-C4 } & $\sigma$ & 1.97168 & $\mathrm{C} 2-\mathrm{N} 10$ & $\sigma^{*}$ & 0.01298 & 2.98 & 1.03 & 0.050 \\
\hline & $\sigma$ & 1.97168 & C3-N10 & $\sigma^{*}$ & 0.01771 & 2.23 & 1.28 & 0.048 \\
\hline & $\sigma$ & 1.97168 & C4-C5 & $\sigma^{*}$ & 0.02151 & 2.21 & 1.23 & 0.047 \\
\hline & $\sigma$ & 1.97168 & C4-C7 & $\sigma^{*}$ & 0.02205 & 2.27 & 1.23 & 0.047 \\
\hline C3-N10 & $\sigma$ & 1.98849 & $\mathrm{C} 3-\mathrm{C} 4$ & $\sigma^{*}$ & 0.03966 & 2.77 & 1.33 & 0.055 \\
\hline \multirow[t]{3}{*}{ C3-N10 } & $\sigma$ & 1.93659 & C2-H14 & $\sigma^{*}$ & 0.01479 & 2.86 & 0.78 & 0.043 \\
\hline & $\sigma$ & 1.93659 & $\mathrm{C} 2-\mathrm{H} 21$ & $\sigma^{*}$ & 0.02002 & 3.53 & 0.77 & 0.047 \\
\hline & $\sigma$ & 1.93659 & C4-C7 & $\sigma^{*}$ & 0.37095 & 7.54 & 0.34 & 0.049 \\
\hline \multirow[t]{4}{*}{ C4-C5 } & $\sigma$ & 1.97274 & $\mathrm{C} 3-\mathrm{C} 4$ & $\sigma^{*}$ & 0.03966 & 2.46 & 1.16 & 0.048 \\
\hline & $\sigma$ & 1.97274 & C3-N11 & $\sigma^{*}$ & 0.04973 & 2.55 & 1.12 & 0.048 \\
\hline & $\sigma$ & 1.97274 & C4-C7 & $\sigma^{*}$ & 0.02205 & 3.83 & 1.25 & 0.062 \\
\hline & $\sigma$ & 1.97274 & C5-C6 & $\sigma^{*}$ & 0.01431 & 2.35 & 1.27 & 0.047 \\
\hline \multirow[t]{5}{*}{ C4-C7 } & $\sigma$ & 1.97515 & $\mathrm{C} 3-\mathrm{C} 4$ & $\sigma^{*}$ & 0.03966 & 2.28 & 1.17 & 0.046 \\
\hline & $\sigma$ & 1.97515 & C3-N10 & $\sigma^{*}$ & 0.01771 & 2.05 & 1.31 & 0.046 \\
\hline & $\sigma$ & 1.97515 & C4-C5 & $\sigma^{*}$ & 0.02151 & 3.79 & 1.26 & 0.062 \\
\hline & $\sigma$ & 1.97515 & C7-C9 & $\sigma^{*}$ & 0.01479 & 2.59 & 1.27 & 0.051 \\
\hline & $\sigma$ & 1.97515 & C9-H19 & $\sigma^{*}$ & 0.01209 & 2.16 & 1.17 & 0.045 \\
\hline \multirow[t]{3}{*}{ C4-C7 } & $\sigma$ & 1.65029 & C3-N10 & $\sigma^{*}$ & 0.23563 & 16.97 & 0.30 & 0.065 \\
\hline & $\Sigma$ & 1.65029 & C5-C6 & $\sigma^{*}$ & 0.29661 & 19.17 & 0.29 & 0.067 \\
\hline & $\sigma$ & 1.65029 & C8-C9 & $\sigma^{*}$ & 0.32715 & 19.81 & 0.28 & 0.067 \\
\hline \multirow[t]{4}{*}{ C5-C6 } & $\sigma$ & 1.98034 & $\mathrm{C} 3-\mathrm{C} 4$ & $\sigma^{*}$ & 0.03966 & 3.50 & 1.52 & 0.065 \\
\hline & $\sigma$ & 1.98034 & C4-C5 & $\sigma^{*}$ & 0.02151 & 2.82 & 1.27 & 0.053 \\
\hline & $\Sigma$ & 1.98034 & C6-C8 & $\sigma^{*}$ & 0.01603 & 2.52 & 1.27 & 0.050 \\
\hline & $\sigma$ & 1.98034 & C8-H17 & $\sigma^{*}$ & 0.01217 & 2.29 & 1.17 & 0.046 \\
\hline \multirow[t]{2}{*}{ C5-C6 } & $\sigma$ & 1.65967 & C4-C7 & $\sigma^{*}$ & 0.37095 & 19.94 & 0.28 & 0.067 \\
\hline & $\sigma$ & 1.65967 & C8-C9 & $\sigma^{*}$ & 0.32715 & 21.05 & 0.28 & 0.069 \\
\hline \multirow[t]{2}{*}{ C5-H15 } & $\sigma$ & 1.98061 & C4-C7 & $\sigma^{*}$ & 0.02205 & 4.31 & 1.08 & 0.061 \\
\hline & $\sigma$ & 1.98061 & C6-C8 & $\sigma^{*}$ & 0.01603 & 3.65 & 1.09 & 0.056 \\
\hline \multirow[t]{3}{*}{ C6-C8 } & $\sigma$ & 1.98120 & C5-C6 & $\sigma^{*}$ & 0.01431 & 2.54 & 1.28 & 0.051 \\
\hline & $\sigma$ & 1.98120 & C5-H15 & $\sigma^{*}$ & 0.01359 & 2.31 & 1.19 & 0.047 \\
\hline & $\sigma$ & 1.98120 & C8-C9 & $\sigma^{*}$ & 0.01572 & 2.43 & 1.27 & 0.050 \\
\hline C6-H16 & $\sigma$ & 1.98266 & $\mathrm{C} 4-\mathrm{C} 5$ & $\sigma^{*}$ & 0.02151 & 3.80 & 1.09 & 0.058 \\
\hline
\end{tabular}


Continued

\begin{tabular}{|c|c|c|c|c|c|c|c|c|}
\hline & $\sigma$ & 1.98266 & C8-C9 & $\sigma^{*}$ & 0.11572 & 3.54 & 1.10 & 0.056 \\
\hline \multirow[t]{2}{*}{ C7-C9 } & $\sigma$ & 1.97999 & $\mathrm{C} 3-\mathrm{C} 4$ & $\sigma^{*}$ & 0.03966 & 3.45 & 1.17 & 0.057 \\
\hline & $\sigma$ & 1.97999 & C4-C7 & $\sigma^{*}$ & 0.02205 & 3.09 & 1.27 & 0.056 \\
\hline \multirow[t]{2}{*}{ C7-H18 } & $\sigma$ & 1.98179 & C4-C5 & $\sigma^{*}$ & 0.02151 & 4.07 & 1.10 & 0.060 \\
\hline & $\sigma$ & 1.98179 & C8-C9 & $\sigma^{*}$ & 0.01572 & 3.51 & 1.10 & 0.056 \\
\hline \multirow[t]{3}{*}{ C8-C9 } & $\sigma$ & 1.98101 & C6-C8 & $\sigma^{*}$ & 0.01603 & 2.42 & 1.27 & 0.049 \\
\hline & $\sigma$ & 1.98101 & C7-C9 & $\sigma^{*}$ & 0.01476 & 2.59 & 1.27 & 0.051 \\
\hline & $\sigma$ & 1.98101 & C7-H18 & $\sigma^{*}$ & 0.01312 & 2.35 & 1.17 & 0.047 \\
\hline \multirow[t]{2}{*}{ C8-C9 } & $\sigma$ & 1.66151 & C4-C7 & $\sigma^{*}$ & 0.37095 & 20.44 & 0.28 & 0.068 \\
\hline & $\sigma$ & 1.66151 & C5-C6 & $\sigma^{*}$ & 0.29661 & 18.58 & 0.29 & 0.066 \\
\hline \multirow[t]{2}{*}{ C8-H17 } & $\sigma$ & 1.98315 & C5-C6 & $\sigma^{*}$ & 0.01431 & 3.50 & 1.11 & 0.056 \\
\hline & $\sigma$ & 1.98315 & C7-C9 & $\sigma^{*}$ & 0.01476 & 3.60 & 1.10 & 0.056 \\
\hline \multirow[t]{2}{*}{ C9-H19 } & $\sigma$ & 1.98276 & C4-C7 & $\sigma^{*}$ & 0.02205 & 3.78 & 1.09 & 0.057 \\
\hline & $\sigma$ & 1.98276 & C6-C8 & $\sigma^{*}$ & 0.01603 & 3.53 & 1.10 & 0.056 \\
\hline \multirow[t]{2}{*}{ N11-H12 } & $\sigma$ & 1.98046 & C3-N10 & $\sigma^{*}$ & 0.01771 & 2.62 & 1.25 & 0.051 \\
\hline & $\sigma$ & 1.98046 & C3-N10 & $\sigma^{*}$ & 0.01771 & 2.14 & 0.69 & 0.036 \\
\hline \multicolumn{9}{|l|}{ LP } \\
\hline \multirow[t]{2}{*}{ N10(1) } & $\sigma$ & 1.91978 & $\mathrm{C} 1-\mathrm{C} 2$ & $\sigma^{*}$ & 0.02495 & 4.86 & 0.70 & 0.053 \\
\hline & $\sigma$ & 1.91978 & C3-N11 & $\sigma^{*}$ & 0.04973 & 11.92 & 0.78 & 0.087 \\
\hline \multirow[t]{3}{*}{ N11(1) } & $\sigma$ & 1.81255 & C1-H13 & $\sigma^{*}$ & 0.02044 & 4.37 & 0.74 & 0.053 \\
\hline & $\sigma$ & 1.81255 & $\mathrm{C} 1-\mathrm{H} 20$ & $\sigma^{*}$ & 0.01525 & 3.21 & 0.75 & 0.046 \\
\hline & $\sigma$ & 1.81255 & C3-N10 & $\sigma^{*}$ & 0.23563 & 29.04 & 0.34 & 0.090 \\
\hline C4-C7 & $\sigma^{*}$ & 0.37095 & C3-N10 & $\sigma^{*}$ & 0.23563 & 118.65 & 0.02 & 0.070 \\
\hline \multirow[t]{2}{*}{ C5-C6 } & $\sigma^{*}$ & 0.29661 & C5(4) & $\pi^{*}$ & 0.00043 & 2.09 & 0.87 & 0.099 \\
\hline & $\sigma^{*}$ & 0.29661 & C6(4) & $\pi^{*}$ & 0.00037 & 2.13 & 0.72 & 0.091 \\
\hline
\end{tabular}

${ }^{\mathrm{a}} \mathrm{E}^{(2)}$ means energy of hyper conjugative interaction (stabilization energy). ${ }^{b}$ Energy difference between donor and acceptor i and $j \mathrm{NBO}$ orbitals. ${ }^{c} \mathrm{~F}(\mathrm{i}, \mathrm{j})$ is the Fock matrix element between $\mathrm{i}$ and $\mathrm{j}$ NBO orbitals.

$$
\begin{gathered}
\Delta \alpha=2^{-1 / 2}\left[\left(\alpha_{x x}-\alpha_{y y}\right)^{2}+\left(\alpha_{y y}-\alpha_{x x}\right)^{2}+6 \alpha_{x x}^{2}\right]^{1 / 2} \\
\beta=\left(\beta_{x}^{2}+\beta_{y}^{2}+\beta_{z}^{2}\right)^{1 / 2}
\end{gathered}
$$

and

$$
\begin{aligned}
& \beta_{x}=\beta_{x x x}+\beta_{x y y}+\beta_{x z z} \\
& \beta_{y}=\beta_{y y y}+\beta_{x x y}+\beta_{y z z} \\
& \beta_{z}=\beta_{z z z}+\beta_{x x z}+\beta_{y y z}
\end{aligned}
$$

The calculated first hyperpolarizability of the $2 \mathrm{PI}$ using $\mathrm{HF} / 6-31 \mathrm{G}(\mathrm{d}, \mathrm{p})$ is $1.0043389 \times 10^{-30} \mathrm{esu}$ and the dipole moment is 5.70065 Debye shown in Table 5 and Table 6. The calculated first hyperpolarizability of 2PI is 14 times greater than the hyperpolarizability of urea. This shows that the $2 \mathrm{PI}$ is useful as a non 
Table 5. The calculated quantum chemical parameters for 2-phenyl-2-imidazoline obtained by B3LYP/6-311++G** calculations.

\begin{tabular}{cc}
\hline Property & 2-phenyl-2-imidazoline \\
\hline Total energy $(\mathrm{eV})$ & -12401.75816 \\
$E_{\mathrm{HOMO}}(\mathrm{eV})$ & -5.82160670 \\
$E_{\mathrm{LUMO}}(\mathrm{eV})$ & -0.78749789 \\
$E_{\mathrm{HOMO}}-E_{\mathrm{LUMO}}(\mathrm{eV})$ & 5.03410881 \\
Electronagativity $(\chi) \mathrm{eV}$ & 3.304552295 \\
Chemical hardness $(\eta) \mathrm{eV}$ & -2.517054405 \\
Electrofilicity index $(\omega) \mathrm{eV}$ & -2.1692153035 \\
Global Softness $(\sigma) \mathrm{eV}$ & -0.3972897836 \\
Total energy change $\left(\Delta E_{T}\right) \mathrm{eV}$ & 0.62926360125 \\
Dipole moment $(D)$ & 5.70065 \\
\hline
\end{tabular}

Table 6. The electric dipole moment $\mu(\mathrm{D})$ the average polarizability $\alpha_{\mathrm{tot}}\left(\times 10^{-12} \mathrm{esu}\right)$ and the first hyperpolarizability $\beta_{\text {tot }}\left(\times 10^{-30} \mathrm{esu}\right)$ of 2-phenyl-2-imidazoline by HF/6-311++G $\mathrm{G}^{\star *}$ method.

\begin{tabular}{cccc}
\hline $\begin{array}{c}\mu \text { and } \boldsymbol{\alpha} \\
\text { components }\end{array}$ & HF/6-31G(d,p) & $\beta$ components & HF/6-31G(d,p) \\
\hline$\mu_{x}$ & 0.104085 & $\beta_{x x x}$ & -91.4409918 \\
$\mu_{y}$ & 0.2171718 & $\beta_{x x y}$ & -14.7087352 \\
$\mu_{z}$ & -1.065635 & $\beta_{x y y}$ & -44.654681 \\
$\mu(D)$ & 1.19357523 & $\beta_{y y y}$ & 0.6127935 \\
$\alpha_{x x}$ & 143.1369019 & $\beta_{x x z}$ & -65.4861453 \\
$\alpha_{x y}$ & 4.2163498 & $\beta_{x y z}$ & 17.3286849 \\
$\alpha_{y y}$ & 56.0399983 & $\beta_{y y z}$ & -2.0474112 \\
$\alpha_{x z}$ & -6.356907 & $\beta_{x z z}$ & 56.4680477 \\
$\alpha_{y z}$ & 17.5520362 & $\beta_{y z z}$ & 11.6736082 \\
$\alpha_{z z}$ & 96.5295611 & $\beta_{z z z}$ & -17.1315722 \\
$\alpha(\mathrm{esu})$ & $14.607899 \times 10^{-12}$ esu & $\beta$ total (esu) & $1.0043389 \times 10^{-30} \mathrm{esu}$ \\
\hline
\end{tabular}

linear optical material.

\section{Electronic Absorption Spectra}

Both the highest occupied molecular orbital (HOMO) and lowest unoccupied molecular orbital (LUMO) are the main orbitals take part in chemical stability [35]. The chemical activity of the molecule is revealed from the HOMO-LUMO energy gap. The HOMO stands for the ability to donate an electron and the LUMO stands for the ability to accept an electron. The HOMO-LUMO energy gap and other molecular properties of 2PI calculated at the DFT/B3LYP method with $6-311++G^{* *}$ level is shown in Table 5. The atomic orbital components of the frontier molecular orbital (HOMO-MO: 39, LUMO-MO: 40) and the Visualization of the molecular orbitals [MO: 32-MO: 47] of 2-phenyl-2-imidazoline under $\mathrm{C}_{1}$ symmetry are shown in Figure 5 and Figure 6 respectively. 


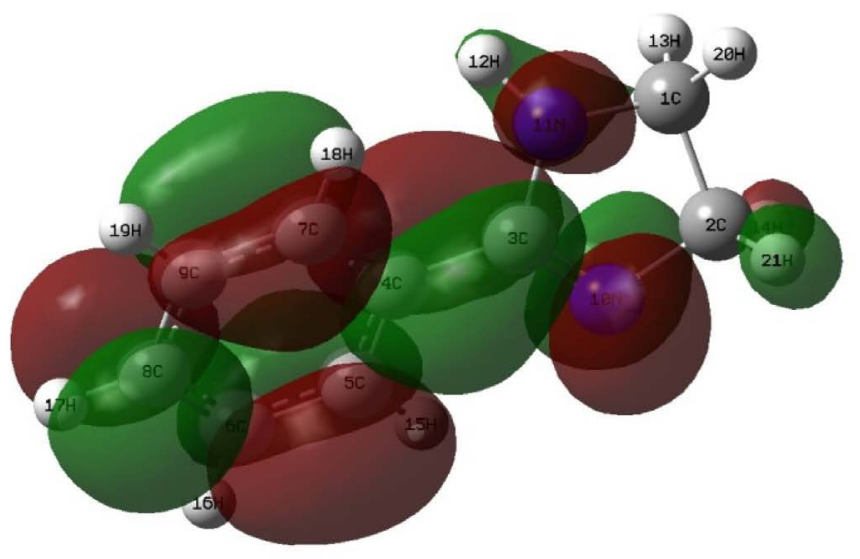

LUMO: $-0.78749789 \mathrm{eV}$

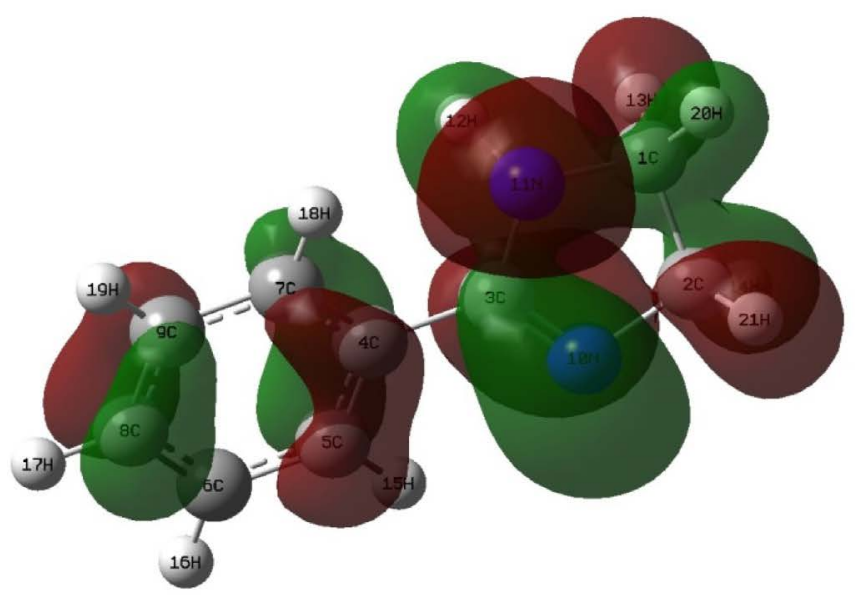

$\Delta \mathrm{E}=5.03410881$

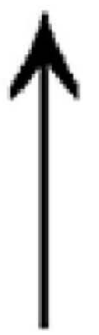

HOMO: $-5.82160670 \mathrm{eV}$

Figure 5. The atomic orbital components of the frontier molecular orbital (HOMO-MO: 39, LUMO-MO: 40) of 2-phenyl-2-imidazoline.

The UV-Visible absorption spectrum of the title molecule using SAC $\mathrm{CI} / 6-311++\mathrm{G}^{* *}$ basis set is performed to determine the lower lying excited states. The computed results involving the vertical excitation energies, oscillator strength ( $f$ ) and wavelength are carried out and compared with measured investigational wavelength. These are presented in Table 7. In accordance with Frank-Condon Principle, the maximum absorption peak correspond in UV-vis spectrum to vertical excitation were carried out SAC-CI method and calculated one strong electronic transition at $272.60 \mathrm{~nm}$ with an oscillator strength $f=$ 0.0658 , shows good agreement with measured experimental data $(\exp =273 \mathrm{~nm})$ as shown in Figure 7. The addition of strong electron-withdrawing C-N substituent at the 2-position not only leads to a large red shift in Imidazoline UV-Vis absorption spectra but it also increases the light absorption efficiency of compound, owing to the facilitation of intramolecular charge transfer (ICT).

Molecular electrostatic potential (MEP) mapping is very useful in the investigation of the molecular structure with its physiochemical property relationships 


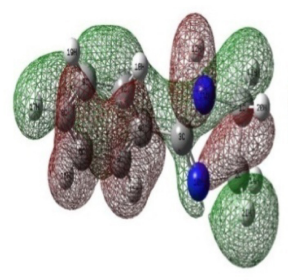

32

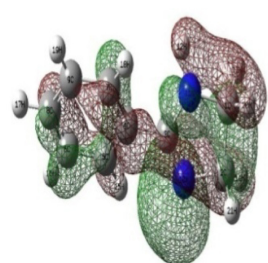

36

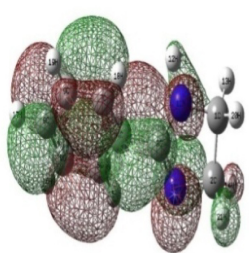

40(LUMO)

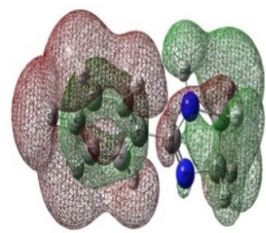

44

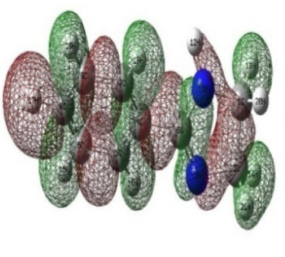

33

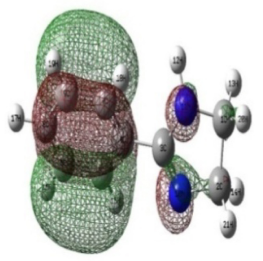

37

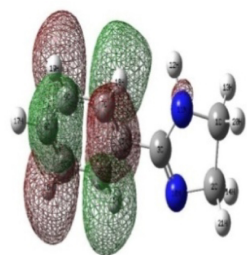

41

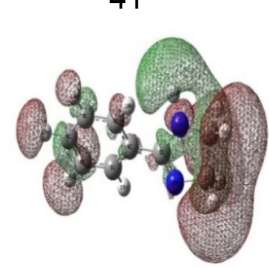

45

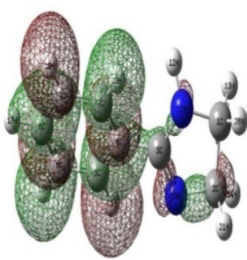

34

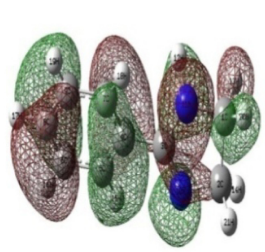

38

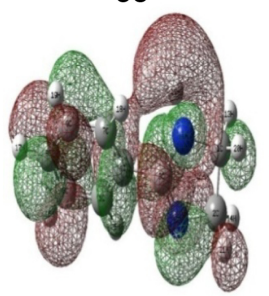

42

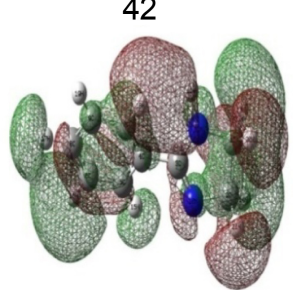

46

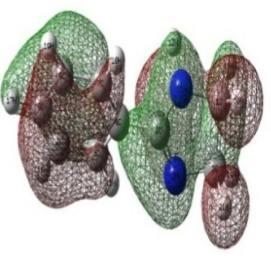

35

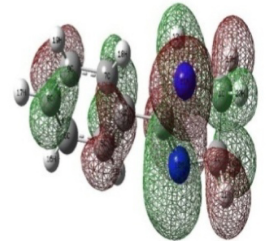

39(HOMO)

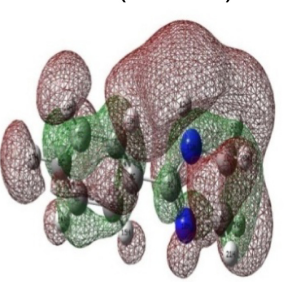

43

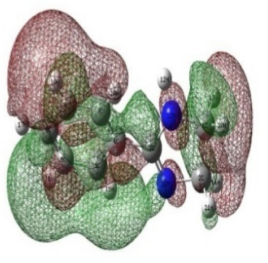

47

Figure 6. Visualization of the molecular orbitals [MO: 32-MO: 47] of 2-phenyl-2-imidazoline under $\mathrm{C}_{1}$ symmetry: HOMO-MO: 39 and LUMO-MO: 40.

[36]-[41]. The significance of MEPs is that it concurrently shows molecular shape, size in addition to positive, negative and neutral electrostatic potential areas. The Molecular electrostatic potential (MEP) mapping of the title compound was shown in Figure 8. MEP is very useful in research of molecular structure with its physicochemical property relationship [42] [43]. The molecular electrostatic potential $V(r)$ at a position $\mathrm{r}$ because of a molecular structure having charges of the nuclei $\left\{Z_{A}\right\}$ positioned at point $\left\{R_{A}\right\}$ and the electron density $\rho(r)$ is specified by

$$
V(r)=\sum_{A}^{N}\left[\left(Z_{A} /\left|r-R_{A}\right|-\int \rho\left(r^{\prime}\right) \mathrm{d}^{3} r^{\prime} /\left|r-r^{\prime}\right|\right)\right]
$$

\section{Thermodynamic Properties}

On the basis of vibrational analyses and statistical thermodynamics, the standard thermodynamic functions: heat capacity, internal energy, entropy and enthalpy were calculated using Moltranv. 2.5 [44] and are listed in Table 8. It is observed 
Table 7. The UV-vis excitation energy and oscillator strength for 2-phenyl-2-imidazalone calculated by SAC CI method.

\begin{tabular}{|c|c|c|c|c|c|c|}
\hline No. & $\begin{array}{c}\text { Exp. } \\
\text { Wavelength }\end{array}$ & Energy $\left(\mathrm{cm}^{-1}\right)$ & $\begin{array}{l}\text { Wavelength } \\
\quad(\mathrm{nm})\end{array}$ & $\begin{array}{l}\text { Osc. } \\
\text { Strength }\end{array}$ & Symmetry & Major contrib. \\
\hline 1 & 273.00 & $36,683.15536$ & 272.60 & 0.0658 & Singlet-A & HOMO- > LUMO (92\%) H-1- > LUMO (5\%) \\
\hline 2 & -- & $41,541.06624$ & 240.72 & 0.0214 & Singlet-A & $\begin{array}{c}\text { H-3- > LUMO (86\%) H-1- > LUMO (7\%), } \\
\text { HOMO- > LUMO }(-3 \%)\end{array}$ \\
\hline 3 & -- & $49,875.25072$ & 200.50 & 0.0059 & Singlet-A & $\begin{array}{c}\mathrm{H}-3->\mathrm{L}+1(90 \%) \mathrm{H}-2->\mathrm{L}+1(-3 \%) \\
\mathrm{H}-1->\mathrm{L}+1(6 \%)\end{array}$ \\
\hline 4 & -- & $58,519.15424$ & 170.88 & 0.0015 & Singlet-A & H-5- > LUMO (97\%) \\
\hline 5 & -- & $62,270.4648$ & 160.58 & 0.0074 & Singlet-A & $\begin{array}{c}\text { HOMO- > L }+4(83 \%) \mathrm{H}-4->\mathrm{LUMO}(-3 \%) \\
\text { H-3- > } \mathrm{L}+2(-2 \%), \mathrm{H}-1->\mathrm{L}+3(-3 \%) \\
\text { HOMO- }>\mathrm{L}+5(-2 \%)\end{array}$ \\
\hline 6 & -- & $65,521.70816$ & 152.62 & 0.0089 & Singlet-A & $\begin{array}{c}\text { HOMO- > L + } 5(72 \%) \mathrm{H}-4->\mathrm{LUMO}(3 \%) \\
\mathrm{H}-2->\mathrm{L}+3(-3 \%), \mathrm{H}-1->\mathrm{L}+2(8 \%) \\
\text { HOMO- > L }+4(2 \%)\end{array}$ \\
\hline 7 & -- & $67,402.60608$ & 148.36 & 0.0352 & Singlet-A & $\mathrm{H}-3->\mathrm{L}+3(81 \%) \mathrm{H}-1->\mathrm{L}+4(-5 \%)$ \\
\hline 8 & -- & $68,145.44784$ & 146.74 & 0.0067 & Singlet-A & $\begin{array}{c}\text { H-7- > LUMO }(82 \%) \text { H-9- > LUMO (6\%), } \\
\text { HOMO- > L + 6 (-2\%) }\end{array}$ \\
\hline 9 & -- & $69,112.51328$ & 144.69 & 0.0245 & Singlet-A & $\begin{array}{c}\mathrm{H}-1->\mathrm{L}+4(60 \%), \text { HOMO- }>\mathrm{L}+6(13 \%) \mathrm{H}-8-> \\
\text { LUMO }(2 \%), \mathrm{H}-3->\mathrm{L}+3(3 \%), \\
\mathrm{H}-1->\mathrm{L}+3(-7 \%)\end{array}$ \\
\hline
\end{tabular}

Table 8. Thermo dynamical properties of 2-phenyl-2-imidazoline obtained by 6-311++ $\mathrm{G}^{\star *}$ density functional calculations.

\begin{tabular}{ccccccc}
\hline $\begin{array}{c}\text { Temperature } \\
(\mathrm{K})\end{array}$ & $C_{V}(\mathrm{~J} / \mathrm{K} / \mathrm{mol})$ & $\begin{array}{c}C_{P} \\
(\mathrm{~J} / \mathrm{K} / \mathrm{mol})\end{array}$ & $\begin{array}{c}U \\
(\mathrm{~J} / \mathrm{K} / \mathrm{mol})\end{array}$ & $\begin{array}{c}H \\
(\mathrm{~J} / \mathrm{K} / \mathrm{mol})\end{array}$ & $\begin{array}{c}S \\
(\mathrm{~J} / \mathrm{K} / \mathrm{mol})\end{array}$ & $\begin{array}{c}G \\
(\mathrm{~J} / \mathrm{K} / \mathrm{mol})\end{array}$ \\
\hline 50 & 41.711 & 50.025 & 463.417 & 463.833 & 257.095 & 450.978 \\
100 & 58.629 & 66.944 & 465.937 & 466.768 & 297.116 & 437.057 \\
150 & 74.786 & 83.100 & 469.266 & 470.513 & 327.267 & 351.423 \\
200 & 94.048 & 102.363 & 473.468 & 475.131 & 353.712 & 304.388 \\
250 & 117.122 & 125.436 & 478.732 & 480.811 & 378.975 & 386.067 \\
300 & 142.556 & 150.871 & 485.223 & 487.717 & 404.079 & 366.493 \\
350 & 168.474 & 176.789 & 492.989 & 495.899 & 429.273 & 345.654 \\
400 & 193.457 & 201.772 & 502.040 & 505.366 & 454.524 & 323.557 \\
500 & 216.713 & 225.028 & 512.313 & 516.055 & 479.662 & 300.207 \\
\hline
\end{tabular}

that the values of $C_{P}, C_{V}, U, H$ and $S$ increase with increase of temperature from 100 to $500 \mathrm{~K}$. The thermo dynamical parameters were fitted with the temperature by quadratic, linear and quadratic formulas respectively.

Further thermo dynamical data provides the needful information about the molecule. Thermo dynamical data used to estimate directions of chemical reactions according second law of thermodynamics in the thermo chemical field. 


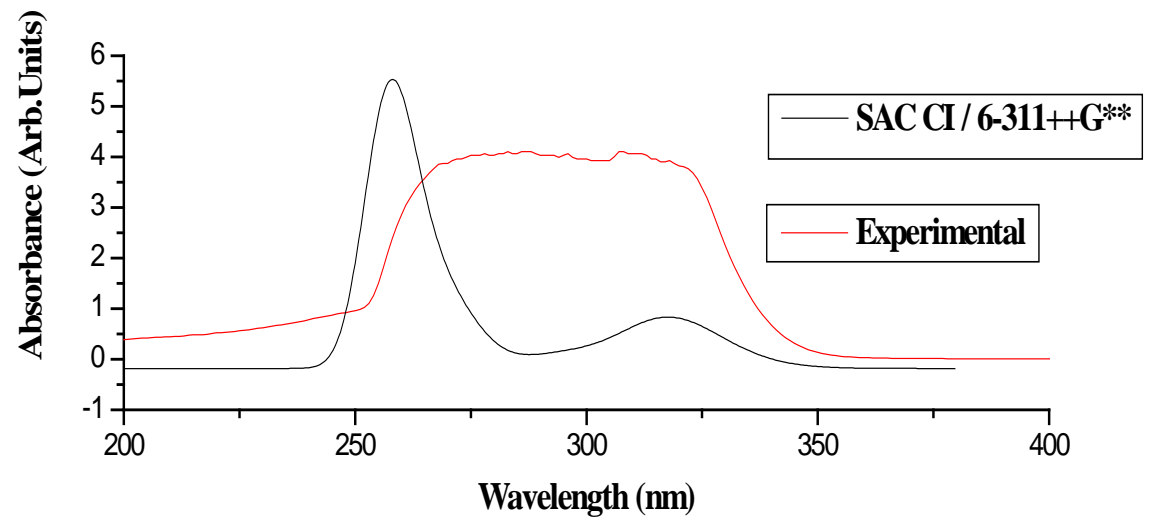

Figure 7. Theoretical and experimental UV/Vis spectra of 2-phenyl-2-imidazoline.

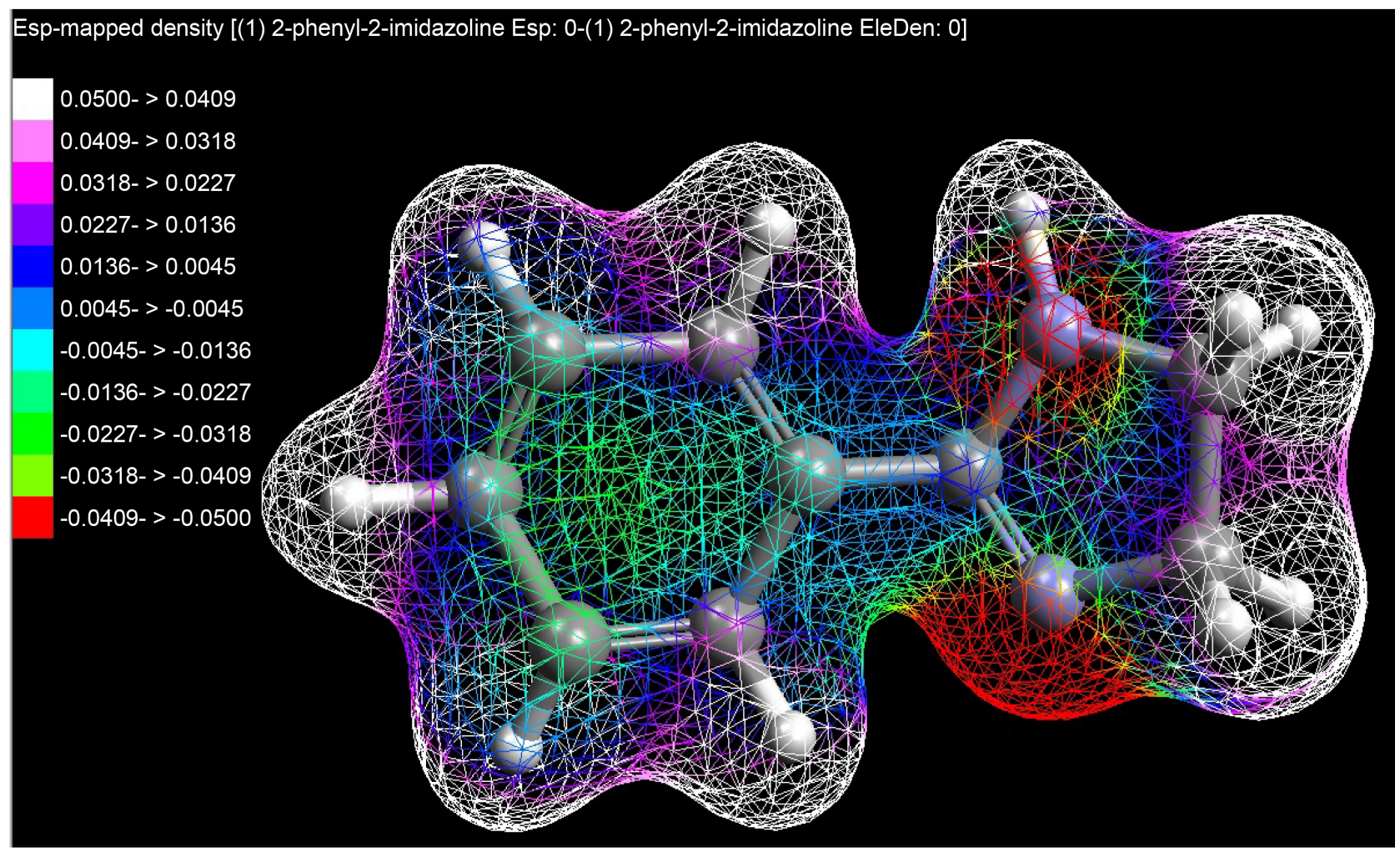

Figure 8. B3LYP/6-311++ $\mathrm{G}^{* *}$ calculated 3D molecular electrostatic potential maps of 2-phenyl-2-imidazoline.

\section{Conclusions}

The theoretical and experimental FT-IR, FT-Raman spectral studies and NBO analysis of $2 \mathrm{PI}$ were carried out and reported. Complete vibrational analysis of 2PI was performed on the basis of DFT calculations at the B3LYP/6-311++ $\mathrm{G}^{* *}$ level of theory and presented. The 42 normal modes of vibrations were ambiguously assigned based on the result of PED output obtained from normal coordinate analysis. There was a qualitative agreement among the calculated and observed frequencies. The NBO analysis shows strong intermolecular hyperconjugative interactions of electron. The strong delocalization of electrons in the molecule is primary to a stabilization of the molecule. 


\section{Acknowledgements}

The First author, Y. Sushma Priya is thankful to Sophisticated Analytical Instrumentation Facility (SAIF), IIT Madras, Chennai and Analytical Chemistry laboratories for their services. The corresponding author, A. Veeraiah is highly grateful to Science and Engineering Research Board, Department of Science and Technology, Government of India for the financial assistance provided. Further, the authors are highly grateful to Prof. T. Sundius for Molvib program.

\section{References}

[1] Vizi, E.S. (1986) Medicinal Research Reviews, 6, 431-449. https://doi.org/10.1002/med.2610060403

[2] Le Bihan, G., Rondu, F., Pele-Tounian, A., Wang, X., Lidy, S., Touboul, E., Lamouri, A., Dive, G., Huet, J., Pfeiffer, B., Renard, P., Guardiola-Lemaitre, B., Manechez, D., Penicaud, L., Ktorza, A. and Godfroid, J.-J. (1999) Journal of Medicinal Chemistry, 42, 1587-1603. https://doi.org/10.1021/jm981099b

[3] Li, H.Y., Drummond, S., De Lucca, I. and Boswell, G.A. (1996) Tetrahedron, 52, 11153-11162. https://doi.org/10.1016/0040-4020(96)00578-9

[4] Ueno, M., Imaizumi, K., Sugita, T., Takata, I. and Takeshita, M. (1995) International Journal of Immunopharmacology, 17, 597-603. https://doi.org/10.1016/0192-0561(95)00057-9

[5] Schorderet, M. (1992) Pharmacologie: Des Concepts Fondamentaux aux Applications the Rapeutiques. Frison-Roche, Paris, 130-153.

[6] Rodríguez-Valdez, L.M., Villamisar, W., Casales, M., González-Rodriguez, J.G., Martínez-Villafañe, A., Martinez, L. and Glossman-mitnik, D. (2006) Corrosion Science, 48, 4053-4064. https://doi.org/10.1016/j.corsci.2006.05.036

[7] González-Rodríguez, C.A., Rodríguez-Gómez, F.J. and Genescá-Llongueras, J. (2008) Electrochimica Acta, 54, 86-90. https://doi.org/10.1016/j.electacta.2008.02.119

[8] Contreras, C.D., Montejo, M., Lopez Gonzalez, J.J., Zinczuk, J. and Brandan, S.A. (2011) Journal of Raman Spectroscopy, 42, 108-116.

[9] Frisch, M.J., Trucks, G.W., Schlegel, H.B., Scuseria, G.E., Robb, M.A., Cheeseman, J.R., Scalmani, G., Barone, V., Mennucci, B., Petersson, G.A., Nakatsuji, H., Caricato, M., Li, X., Hratchian, H.P., Izmaylov, A.F., Bloino, J., Zheng, G., Sonnenberg, J.L., Hada, M., Ehara, M., Toyota, K., Fukuda, R., Hasegawa, J., Ishida, M., Nakajima, T., Honda, Y., Kitao, O., Nakai, H., Vreven, T., Montgomery, J.A., Peralta, J.E., Ogliaro, F., Bearpark, M., Heyd, J.J., Brothers, E., Kudin, K.N., Staroverov, V.N., Kobayashi, R., Normand, J., Raghavachari, K., Rendell, A., Burant, J.C., Iyengar, S.S., Tomasi, J., Cossi, M., Rega, N., Millam, J.M., Klene, M., Knox, J.E., Cross, J.B., Bakken, V., Adamo, C., Jaramillo, J., Gomperts, R., Stratmann, R.E., Yazyev, O., Austin, A.J., Cammi, R., Pomelli, C., Ochterski, J.W., Martin, R.L., Morokuma, K., Zakrzewski, V.G., Voth, G.A., Salvador, P., Dannenberg, J.J., Dapprich, S., Daniels, A.D., Farkas, Ö., Foresman, J.B., Ortiz, J.V., Cioslowski, J. and Fox, D.J. (2009) Gaussian. Wallingford.

[10] Dennington, R., Keith, T. and Millam, J. (2009) Gauss View. Version 5, Semichem Inc., Shawnee Mission.

[11] Rauhut, G. and Pulay, P. (1995) The Journal of Physical Chemistry, 99, 3093-3100. https://doi.org/10.1021/j100010a019 
[12] Pulay, P., et al. (1983) Journal of the American Chemical Society, 105, 7037-7047. https://doi.org/10.1021/ja00362a005

[13] Fogarasi, G., Pulay, P. and Durig, J.R. (1985) Chapter 3, Vibrational Spectra and Structure. Elsevier, Amsterdam, Vol. 14, 125.

[14] Sundius, T. (1990) Journal of Molecular Structure, 218, 321-326. https://doi.org/10.1016/0022-2860(90)80287-T

[15] Sundius, T. (2002) Vibrational Spectroscopy, 29, 89-95. https://doi.org/10.1016/S0924-2031(01)00189-8

[16] Glendering, E.D., Reed, A.E., Carpenter, J.E. and Weinhold, F. (1998) NBO Version 3.1. TCI, University of Wisconsin, Madison.

[17] Polavarapu, P.L. (1990) The Journal of Physical Chemistry, 94, 8106-8112. https://doi.org/10.1021/j100384a024

[18] Keresztury, G., Holly, S., Varga, J., Besenyei, G., Wang, A.Y. and Durig, J.R. (1993) Spectrochimica Acta Part A, 49, 2007-2017. https://doi.org/10.1016/S0584-8539(09)91012-1

[19] Becke, A.D. (1993) The Journal of Chemical Physics, 98, 5648-5652. https://doi.org/10.1063/1.464913

[20] Jing, L.-L., Ma, H.-P., He, L., Fan, P.-C. and Jia, Z.-P. (2011) Acta Crystallographica Section E, 67, o3503.

[21] Pulay, P., Fogarasi, G., Pang, F. and Boggs, J.E. (1979) Journal of the American Chemical Society, 101, 2550.

[22] Fogarasi, G., Zhou, X., Taylor, P.W. and Pulay, P. (1992) Journal of the American Chemical Society, 114, 8191-8201. https://doi.org/10.1021/ja00047a032

[23] Lambert, J.B., Shurvell, H.F., Verbit, L., Cooks, R.G. and Stout, G.H. (1976) Organic Structural Analysis. Macmillan Publ. Co. Inc., New York.

[24] Silverstein, R.M., Basseler, G.C. and Morill, C. (1981) Spectrometric Identification of Organic Compounds. John Wiley and Sons, New York.

[25] Veeraiah, A. (2015) Spectrochimica Acta Part A: Molecular and Biomolecular Spectroscopy, 147, 212-224. https://doi.org/10.1016/j.saa.2015.03.053

[26] Chandra, S., Saleem, H., Sebastian, S. and Sundaraganesan, N. (2011) Spectrochimica Acta Part A, 78, 1515-1524. https://doi.org/10.1016/j.saa.2011.01.043

[27] Furic, K., Mohack, V., Bonifacic, M. and Stefanic, I. (1992) Journal of Molecular Structure, 267, 39-44. https://doi.org/10.1016/0022-2860(92)87006-H

[28] Colthup, N.B., Daly, L.H. and Wiberley, S.E. (1990) Introduction to Infrared and Raman Spectroscopy. Academic Press, New York.

[29] Smith, B.C. (1998) Infrared Spectral Interpretation: A Systematic Approach. CRC Press, Boca Raton, FL.

[30] Socrates, G. (2001) Infrared and Raman Characteristic Group Frequencies-Tables and Charts. 3rd Edition, Wiley \& Sons, Chichester.

[31] Nagabalasubramanian, P.B., Periandy, S., Mohan, S. and Govindarajan, M. (2009) Spectrochimica Acta Part A, 73, 277-280. https://doi.org/10.1016/j.saa.2009.02.044

[32] Silverstein, M., Clayton Bassler, G. and Morril, C. (1981) Spectroscopic Identification of Organic Compounds. John Wiley, New York.

[33] James, C., AmalRaj, A., Reghunathan, R., Hubert Joe, I. and JayaKumar, V.S. (2006) Journal of Raman Spectroscopy, 37, 1381-1392. https://doi.org/10.1002/jrs.1554

[34] Na, L.J., Rang, C.Z. and Fang, Y.S. (2005) Journal of Zhejiang University Science B, 
6, 584-589.

[35] Gunasekaran, S., Balaji, R.A., Kumaresan, S., Anand, G. and Srinivasan, S. (2008) Canadian Journal of Analytical Sciences and Spectroscopy, 53, 149-162.

[36] Murray, J.S. and Sen, K. (1996) Molecular Electrostatic Potentials, Concepts and Applications. Elsevier, Amsterdam.

[37] Alkorta, I. and Perez, J.J. (1996) International Journal of Quantum Chemistry, 57, 123-135.

https://doi.org/10.1002/(SICI)1097-461X(1996)57:1<123::AID-QUA14>3.0.CO;2-9

[38] Lowdin, P. (1978) Advances in Quantum Chemistry. Academic Press, New York.

[39] Luque, F.J., Orozco, M., Bhadane, P.K. and Gadre, S.R. (1993) The Journal of Physical Chemistry, 97, 9380-9384. https://doi.org/10.1021/j100139a021

[40] Sponer, J. and Hobza, P. (1996) International Journal of Quantum Chemistry, 57, 959-970.

https://doi.org/10.1002/(SICI)1097-461X(1996)57:5<959::AID-QUA16>3.0.CO;2-S

[41] Anbarasana, P.M., Subramanian, M.K., Senthilkumar, P., Mohanasundaram, C., Ilangovan, V. and Sundaraganesan, N. (2011) Journal of Chemical and Pharmaceutical Research, 3, 597-612.

[42] Murray, J.S. and Sen, K. (1996) Molecular Electrostatic Potentials, Concepts and Applications. Elsevier, Amsterdam.

[43] Scrocco, E. and Tomasi, J. (1978) Electronic Molecular Structure, Reactivity and Intermolecular Forces: An Heuristic Interpretation by Means of Electrostatic Molecular Potentials. In: Lowdin, P.O., Ed., Advances in Quantum Chemistry, Academic Press, New York, p. 115.

[44] Ignatov, S.K. (2004) Program for Molecular Visualization and Thermodynamic Calculations. University of Nizhny Novgorod, Nizhnij Novgorod.

$\underline{\text { http://ichem.unn.ru/Moltran }}$ 


\section{Supplementary Material 1}

Table S1. Definition of internal coordinates of 2-phenyl-2-imidazoline.

\begin{tabular}{|c|c|c|c|}
\hline No.(i) & Symbol & Type & Definition $^{\mathrm{a}}$ \\
\hline \multicolumn{4}{|r|}{ Stretching } \\
\hline $1-8$ & $R_{i}$ & $\mathrm{CC}$ & $\mathrm{C} 1-\mathrm{C} 2, \mathrm{C} 3-\mathrm{C} 4, \mathrm{C} 4-\mathrm{C} 5, \mathrm{C} 5-\mathrm{C} 6, \mathrm{C} 6-\mathrm{C} 8, \mathrm{C} 8-\mathrm{C} 9, \mathrm{C} 4-\mathrm{C} 7, \mathrm{C} 7-\mathrm{C} 9$ \\
\hline $9-12$ & $r_{i}$ & $\mathrm{CN}$ & C1-N11, C2-N10, C3-N11, C3-N10 \\
\hline $13-21$ & $P_{i}$ & $\mathrm{CH}$ & C5-H15, C6-H16, C8-H17, C9-H19, C7-H18, C1-H20, C1-H13, C2-H14, C2-H21. \\
\hline 22 & $Q_{i}$ & $\mathrm{NH}$ & N11-H12. \\
\hline \multicolumn{4}{|r|}{ In-Plane bending } \\
\hline $23-28$ & $\beta_{i}$ & Ring1 & C4-C5-C6, C5-C6-C8, C6-C8-C9, C8-C9-C7, C9-C7-C4, C7-C4-C5. \\
\hline $29-33$ & $\beta_{i}$ & Ring2 & C1-C2-N10, C2-N10-C3, N10-C3-N11, C3-N11-C1, N11-C1-C2. \\
\hline $34-43$ & $\beta_{i}$ & $\mathrm{CCH}$ & $\begin{array}{c}\text { C4-C7-H18, C9-C7-H18, C7-C9-H19, C8-C9-H19, C9-C8-H17, C6-C8-H17, } \\
\text { C8-C6-H16, C5-C6-H16, C4-C5-H15, C6-C5-H115, C1-C2-H14, } \\
\text { N10-C2-H14. }\end{array}$ \\
\hline $44-47$ & $\theta_{i}$ & $\mathrm{CN}$ & C1-N11-H12, C3-N11-H12, N11-C3-C4, N10-C3-C4. \\
\hline $48-49$ & $\theta_{i}$ & $\mathrm{CC}$ & $\mathrm{C} 7-\mathrm{C} 4-\mathrm{C} 3, \mathrm{C} 5-\mathrm{C} 4-\mathrm{C} 3$. \\
\hline $50-53$ & $\theta_{i}$ & $\mathrm{NCH}$ & N11-C1-H13, N11-C1-H20, N10-C2-H14, N10-C2-H21. \\
\hline $54-55$ & $\theta_{i}$ & $\mathrm{HCH}$ & $\mathrm{H} 13-\mathrm{C} 1-\mathrm{H} 20, \mathrm{H} 14-\mathrm{C} 2-\mathrm{H} 21$. \\
\hline \multicolumn{4}{|r|}{ Out-of-plane bending } \\
\hline $56-60$ & $\dot{\omega}_{i}$ & $\mathrm{CH}$ & H15-C5-C4-C6, H16-C6-C5-C8, H17-C8-C6-C9, H19-C9-C8-C7, H18-C7-C9-C4. \\
\hline 61 & $\varphi_{i}$ & $\mathrm{NH}$ & H12-N11-C3-C1 \\
\hline 62 & $\pi_{i}$ & $\mathrm{CN}$ & C4-C3-N10-N11 \\
\hline 63 & $\pi_{i}$ & $\mathrm{CC}$ & $\mathrm{C} 3-\mathrm{C} 4-\mathrm{C} 7-\mathrm{C} 5$ \\
\hline \multicolumn{4}{|r|}{ Torsion } \\
\hline $64-69$ & $\tau_{i}$ & $\tau$ RING1 & $\begin{array}{l}\text { C4-C5-C6-C8, C5-C6-C8-C9, C6-C8-C9-C7, C8-C9-C7-C4, C9-C7-C4-C5, } \\
\text { C7-C4-C5-C6 }\end{array}$ \\
\hline $70-74$ & $\tau_{i}$ & $\tau$ RING2 & C1-C2 -N10-C3, C2-N10-C3-N11, N10-C3-N11-C1, C3-N11-C1-C2, N11-C1-C2-N10 \\
\hline $75-82$ & $\tau_{i}$ & $\tau \mathrm{CH}_{2}$ & $\begin{array}{l}\text { C3-N11-C1-H13, C3-N11-C1-H20, N10-C2-C1-H13, N10-C2-C1-H20, } \\
\text { N11-C1-C2-H14, N11-C1-C2-H21, C3-N10-C2-H14, C3-N10-C2-H21. }\end{array}$ \\
\hline $83-84$ & $\tau_{i}$ & Butterfly & N10-C3-C4-C7, C5-C4-C3-N11. \\
\hline
\end{tabular}

${ }^{\mathrm{a}}$ For numbering of atom refer Figure 1. 